University of Nebraska - Lincoln

DigitalCommons@University of Nebraska - Lincoln

Faculty Publications from the Department of

Electrical and Computer Engineering

Femtosecond Photon-Mediated Plasma Enhances

Photosynthesis of Plasmonic Nanostructures and Their SERS Applications

Peng Ran

Lan Jiang

Xin Li

Bo Li

Pei Zuo

See next page for additional authors

Follow this and additional works at: https://digitalcommons.unl.edu/electricalengineeringfacpub

Part of the Computer Engineering Commons, Electrical and Computer Engineering Commons, Materials Science and Engineering Commons, and the Nanoscience and Nanotechnology Commons

This Article is brought to you for free and open access by the Electrical \& Computer Engineering, Department of at DigitalCommons@University of Nebraska - Lincoln. It has been accepted for inclusion in Faculty Publications from the Department of Electrical and Computer Engineering by an authorized administrator of DigitalCommons@University of Nebraska - Lincoln. 
Authors

Peng Ran, Lan Jiang, Xin Li, Bo Li, Pei Zuo, and Yongfeng Lu 


\title{
Femtosecond Photon-Mediated Plasma Enhances Photosynthesis of Plasmonic Nanostructures and Their SERS Applications
}

\author{
Peng Ran, ${ }^{1}$ Lan Jiang, ${ }^{1}$ Xin Li, ${ }^{\text {* }}$ Bo Li, ${ }^{1}$ Pei Zuo, ${ }^{1}$ \\ and Yongfeng $\mathrm{Lu}^{2}$
}

1 Laser Micro/Nano Fabrication Laboratory, School of Mechanical Engineering, Beijing Institute of Technology, Beijing 100081, P. R. China

2 Department of Electrical and Computer Engineering University of NebraskaLincoln, Lincoln, NE 68588-0511, USA

Corresponding author — Xin Li, email lixin02@bit.edu.cn

\begin{abstract}
Laser ablation in liquid has proven to be a universal and green method to synthesize nanocrystals and fabricate functional nanostructures. This study demonstrates the superiority of femtosecond laser-mediated plasma in enhancing photoredox of metal cations for controllable fabrication of plasmonic nanostructures in liquid. Through employing upstream high energetic plasma during laser-induced microexplosions, single/three-electron photoreduction of metallic cations can readily occur without chemical reductants or capping agents. Experimental evidences demonstrate that this process exhibits higher photon utilization efficiency in yield of colloidal metal nanoparticles than direct irradiation of metallic precursors. Photogenerated hydrated electrons derived from strong ionization of silicon and water are responsible for this enhanced consequences. Furthermore, these metallic nanoparticles are accessible to self-assemble into nanoplates for silver and nanospheres for gold, favored by surface-tension gradients between laser irradiated and unirradiated regions. These metallic nanostructures exhibit excellent surfaceenhanced Raman spectroscopy performance in trace detection of Rhodamine 6G
\end{abstract}

Published in Small 2019, 1804899

DOI: 10.1002/smll.201804899

Copyright (c) 2019 WILEY-VCH Verlag GmbH \& Co. KGaA, Weinheim. Used by permission. Submitted November 20, 2018; revised January 27, 2019. 
(R6G), 4-mercaptobenzoic acid (4-MBA), and mercapto-5-nitrobenzimidazole molecules with high sensitivity (down to $10^{-12} \mathrm{~mol} \mathrm{~L}^{-1}, 30 \times 10^{-15} \mathrm{M}$ for R6G), good reproducibility (relative standard deviation $<7 \%$ ), and good dual-analyte detection ability with mixture ratios of R6G to 4-MBA ranging from 20 to 0.025 . The conceptual importance of this plasma-enhanced-photochemical process may provide exciting opportunities in photochemical reactions, plasmofluidics, and material synthesis.

Keywords: femtosecond laser, metallic nanostructures, photoreduction, plasma, SERS

\section{Introduction}

Plasmonic nanostructures, capable of strong interaction with incident light by exciting localized surface plasmon resonance (LSPR), have attracted numerous interests in the fields of plasmon-enhanced spectroscopies, ${ }^{[1]}$ imaging $_{1}{ }^{[2]}$ chemical transformations, ${ }^{[3]}$ and solar energy conversion. ${ }^{[3, d]}$ Notably, the enhancedelectromagnetic field ("hot spots") near plasmonic surfaces because of strong plasmon resonance coupling fully benefits to magnify the cross-section of surface-enhanced Raman spectroscopy (SERS) ${ }^{[1 b, c, 4]}$ and extended applications. ${ }^{[5]}$ During these years, cited methods for synthesizing plasmonic nanostructures, such as chemical synthesis, ${ }^{[6]}$ photoreduction, ${ }^{[7]}$ and electron beam lithography, ${ }^{[4 d, 8]}$ have been developed to facilitate their applications. Whereas the residual photoinitiators and reducing agents on plasmonic surfaces are always unexpected in biomedical analysis and redox reaction proceeding. To this end, the exploration of high tunable and surfactant-free approaches in fabricating SERS-active nanostructures is still highly in demand.

In recent two decades, laser ablation in liquid (LAL) has been intensively studied as a green, simple, and versatile method to synthesize functional nanomaterials with various compositions and morphologies. ${ }^{[9]}$ Generally, two categories are typically adopted to synthesize plasmonic nanostructures: chemical bottom-up and physical topdown. ${ }^{[9]}$ Photochemical reduction of metal precursors features bottom-up approach to synthesize colloidal nanoparticles. ${ }^{[7 a, b, 9 d, 10]}$ By photolyzing the metal complexes, ${ }^{[11]}$ or by using the photosensitive regents or photochemically generated intermediates (e.g., hydrated electrons and radicals from water radiolysis), ${ }^{[7 a, 10]}$ metal cations could be photoreduced to neutral atoms or clusters. The top-down method mainly depends on laser ablation of bulk into nanoparticle dispersions. 
[12] The main mechanisms are based on converting the laser energy into material plasma or vapor phases, and micro/nano droplets. And they subsequently quench and react with the surrounding mediums to form colloidal nanostructures. ${ }^{[9 b, c, 12 b, 13]}$ From this technique, numerous newfangled nanostructures with excellent plasmonic properties have been successfully synthesized with LAL technique by selecting the appropriate solvents, ligands, and laser parameters, which exhibit huge potential in plasmonic science and technologies. ${ }^{[9 a-d]}$

But for practical SERS detection, colloidal nanoparticles synthesized by LAL can still be challenges for producing large and stable enhancement of Raman signals, due to the discrete and random distribution of hot spots. ${ }^{[1 c]}$ Self-assembly of metallic nanoparticles arrays onto a substrate are usually executed, ${ }^{[4,14]}$ whereas the poor adhesive forces to substrates might restrict their practical applications. Recently, incorporating plasmonic structures onto solid substrates by lasers have been intensively studied for applications on reproducible biochemical analysis and diagnostics due to their on-demand fabrication features, which possess huge potential in practical applications. ${ }^{[4 b, 15]}$ Whereas the metal bulk targets are always involved, ${ }^{[15 b]}$ or the residual photoinitiators and reducing agents during photoreduction/ photopolymerization process may risk lowering signal-to-noise ratio of SERS detections and obstructing some chemical reaction proceeding. ${ }^{[4 b, c, 15 e]}$ From this aspect, controllable engineering SERS platforms with high sensitivity and good attachment to substrates by LAL without any reductants and capping regents still remain challenges.

In this article, we present the significance of femtosecond laser-mediated plasma in enhancing photosynthesis of plasmonic nanostructures. By employing reductive components (electrons, ions) in plasma during laser-induced microexplosion, single/multielectron reduction of metal ions $\left(\mathrm{Ag}^{+}\right.$and $\left.\mathrm{Au}^{3+}\right)$ is realized. This process exhibits higher utilization efficiency of incident photons than direct photoreduction rections, attributing to more photogenerated hydrated electrons. Notably, reducing and capping regents required in chemical synthesis are absent during the whole process, which is particularly significant in biomedical applications. Noteworthily, the irradiated regions are more hydrophilic than the original surfaces, which favors in situ assemble of photoreduced metal nanoparticles into plasmonic nanostructures. These metallic nanostructures exhibit excellent SERS performances in trace detection of Rhodamine 6G (R6G), 4-mercaptobenzoic acid 
(4-MBA), and mercapto-5-nitrobenzimidazole (MNBI) molecules with high sensitivity (down to $10^{-12} \mathrm{~mol} \mathrm{~L}^{-1}$ ), good reproducibility (relative standard deviation, RSD $<7 \%$ ), and excellent dual-analyte detection ability with mixture ratios of R6G to 4-MBA ranging from 20 to 0.025 .

\section{Results and Discussion}

\subsection{Controllable Fabrication of Silver Nanostructures}

Arguably, femtosecond laser, featuring ultrashort irradiation period and ultrahigh intensity, could easily excite abundant free electrons (exceeding $\approx 10^{21} \mathrm{~cm}^{-3}$ ). ${ }^{[16]}$ The high ionization rate (more free electrons) provides much potentials in synthesizing plasmonic structures, compared with vapor or metal droplets generated by long pulse lasers. ${ }^{[13 b]}$ Generally, abundant high energetic particles generate and rapidly disperse into solvents in a strong shockwave when injecting a femtosecond photon flux. Except for the formation of heavy particles (quantum dots, nanoparticles, atom clusters, etc.), featuring slow portion during LAL process, numerous light and/or fast components (ions, electrons, etc.) are also involved and meritorious for some chemical processes. [9b,c,13a] In the present work, we aim at figuring out the potential roles of the fast components in plasma for synthesizing plasmonic nanostructures. The detailed information of the experimental setup is presented in Figure S1 (Supporting Information). As illustrated in Figure 1a, strong excitation of valence electrons in silicon by femtosecond laser leads an adiabatic explosion of plasma plume, which carries massive activated species (electrons, ions, atoms, clusters, etc.), and holds great promise for a designated redox reaction. In the case of $\mathrm{AgNO}_{3^{\prime}}$ reduction of silver cations $\left(\mathrm{Ag}^{+}\right)$occurred at the interface of silicon plasma and liquid, by trapping the upstream electrons during plasma expansion to yield neutral particles $\left(\mathrm{Ag}^{0}\right)$. Meanwhile, laser-induced silicon plasma could excite water plasma to generate hydrated electrons and radicals to further reduce the $\mathrm{Ag}^{+}$into silver atoms. ${ }^{\left[{ }^{[9 d]}\right.}$ Additionally, the photoreduced nanoparticles are simultaneously partially sintered onto rippled silicon as the subsequent growth sites of silver nanostructures by attaching dissociative silver in liquid (Figure 1a2).

Experimentally, the morphologies of plasmonic nanostructures depend significantly on laser energies. As shown in Figure 1c, the 

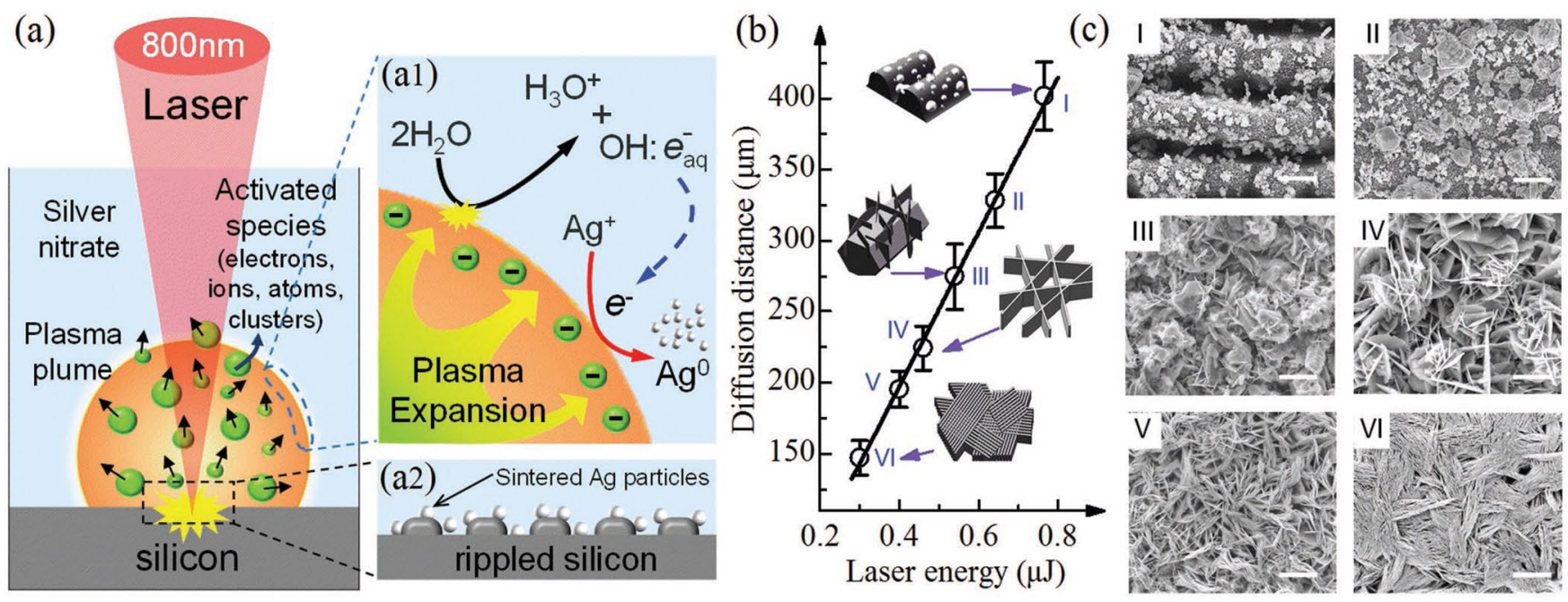

Figure 1. Laser-mediated-plasma reduction of $\mathrm{AgNO} 3$ at the silicon-water interface. a) Schematic illustration of the experiment mechanism: a1) Reduction of $\mathrm{Ag}^{+}$ at the interface of plasma-liquid by trapping upstream electrons during plasma expansion, or by hydrated electrons and radicals from water radiolysis induced by laser excitation or silicon plasma; a2) Formation of growth sites by sintering reduced silver particles onto rippled silicon. b) Laser energy dependence of bubble diffusion distances within a second on irradiation energy of femtosecond laser (other form factors were the same). Insets: Schematic representations of the Ag-nanostructures on silicon surfaces. c) The corresponding SEM images of Ag-nanostructures synthesized under different laser energy of: I-0.76 $\mu \mathrm{J}$, II-0.64 $\mu \mathrm{J}$, III-0.54 $\mu \mathrm{J}$, IV-0.46 $\mu \mathrm{J}$, $\mathrm{V}-0.4 \mu \mathrm{J}$, and $\mathrm{VI}-0.3 \mu \mathrm{J}$, respectively.

corresponding scanning electron microscope (SEM) morphologies are displayed: particles on substrate (I and II), nanoplates on blocks (III), nanoplate clusters (IV), and aligned nanoplates (V and VI). Accordingly, one could readily tune the morphologies and corresponding coverage fractions by controlling the laser energy, and a lower energy intensity contributes significantly to obtaining dense nanostructures. To reveal the potential mechanism, the eruption dynamics of plasma plume have been captured by a commercial charge-coupled device (CCD) (Figure S2, Supporting Information). Arguably, plasma featuring high temperature and high pressure will generate temperature and pressure gradients at the interface of plasma and liquid, ${ }^{[17]}$ and induce Marangoni flow between them. Plasma plume carrying active species explode outward from focused volume to the surroundings due to the established temperature gradient (Marangoni flow), and the diffusion distances of bubbles increase linearly with laser energy (Figure 1b). 
However, energy dispersive X-ray spectroscopy (EDS) demonstrates that the amount of silver element sintered on silicon depends negatively on the incident laser energies (Figure S3, Supporting Information) due to the much faster expansion of shockwave under high energy excitation. In this work, we pay more attention to synthesizing plasmonic nanoplates, which possess sharp edges with high curvature than nanospheres for plasmonic applications. ${ }^{[7 b]}$ Therefore, a femtosecond laser with low energy intensity contributes significantly in obtaining such nanostructures. Purposely modulating laser energy down to $0.5 \mu \mathrm{J}$ or less, dense silver nanoplates (AgNPs) with abundant $\approx 10$ $\mathrm{nm}$ nanogaps mushroom and overspread the whole irradiated areas. Studies on plasmonic metals have well documented that electric field enhancements created at the nanoscale gaps between two adjacent metallic nanostructures are normally two or more orders of magnitude larger than those generated around isolated ones. ${ }^{[8,18]}$ So these closely packed ultrathin AgNPs may offer unreserved opportunities for confining incident electromagnetic radiation to amplify the lightmatter interactions. ${ }^{[4 a, 8]}$ Moreover, Ag nanostructures match well the plasmon resonance condition at visible region, which may provide a handy avenue to tune the LSPR features for plasmonic applications, especially for surface enhanced spectroscopies. ${ }^{[4 a]}$

The fabrication process of closely packed AgNPs has been experimentally explored. Laser ablation of silicon would generate plasma and induce rippled structures. Utilizing the highenergetic plasma, silver cations are massively reduced into nanoparticles, with being partly sintered onto the rippled substrate. As demonstrated in Figure $2 \mathrm{~b}$, Ag atomic clusters (gloss white) are clearly distinguished on Si wafer after irradiation without subsequent growth process, and the corresponding EDS element map demonstrates the existence and distribution of Ag (colored by green, Figure 2h), which will promote the self-assemble and growth of nanoplates due to the pinning effects. ${ }^{[19]}$ Figure $2 b-e$ shows the typical SEM images of as prepared AgNPs at four selected growing times. The stacking densities of AgNPs increase as the growing time is prolonged. The magnified SEM image reveals the thickness of AgNPs is about $40 \pm 10 \mathrm{~nm}$ and the existence of abundant $\approx 10 \mathrm{~nm}$ nanogaps (Figure $2 \mathrm{f}$ ), which fully benefit plasmonic relevant applications. ${ }^{[4,20]}$ By comparison, similar AgNPs arrays are also formed on the substrate with $\approx 130 \mathrm{~nm}$ columnar structures 


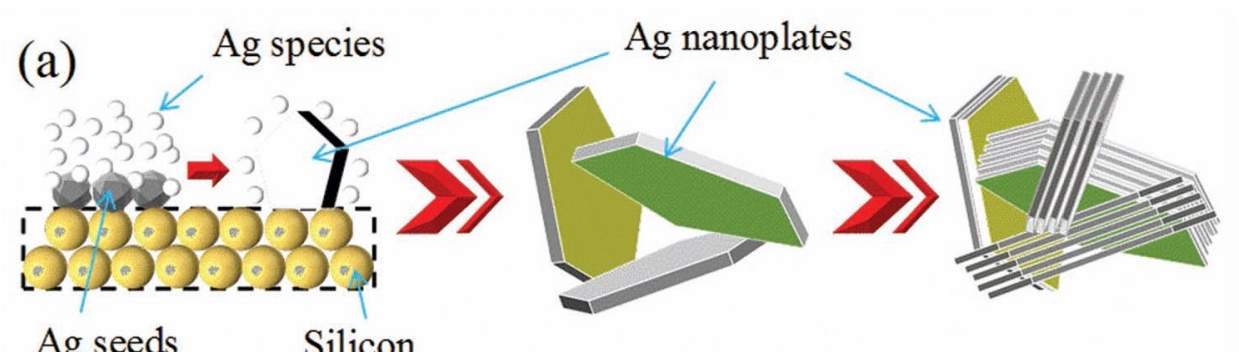

Ag seeds Silicon
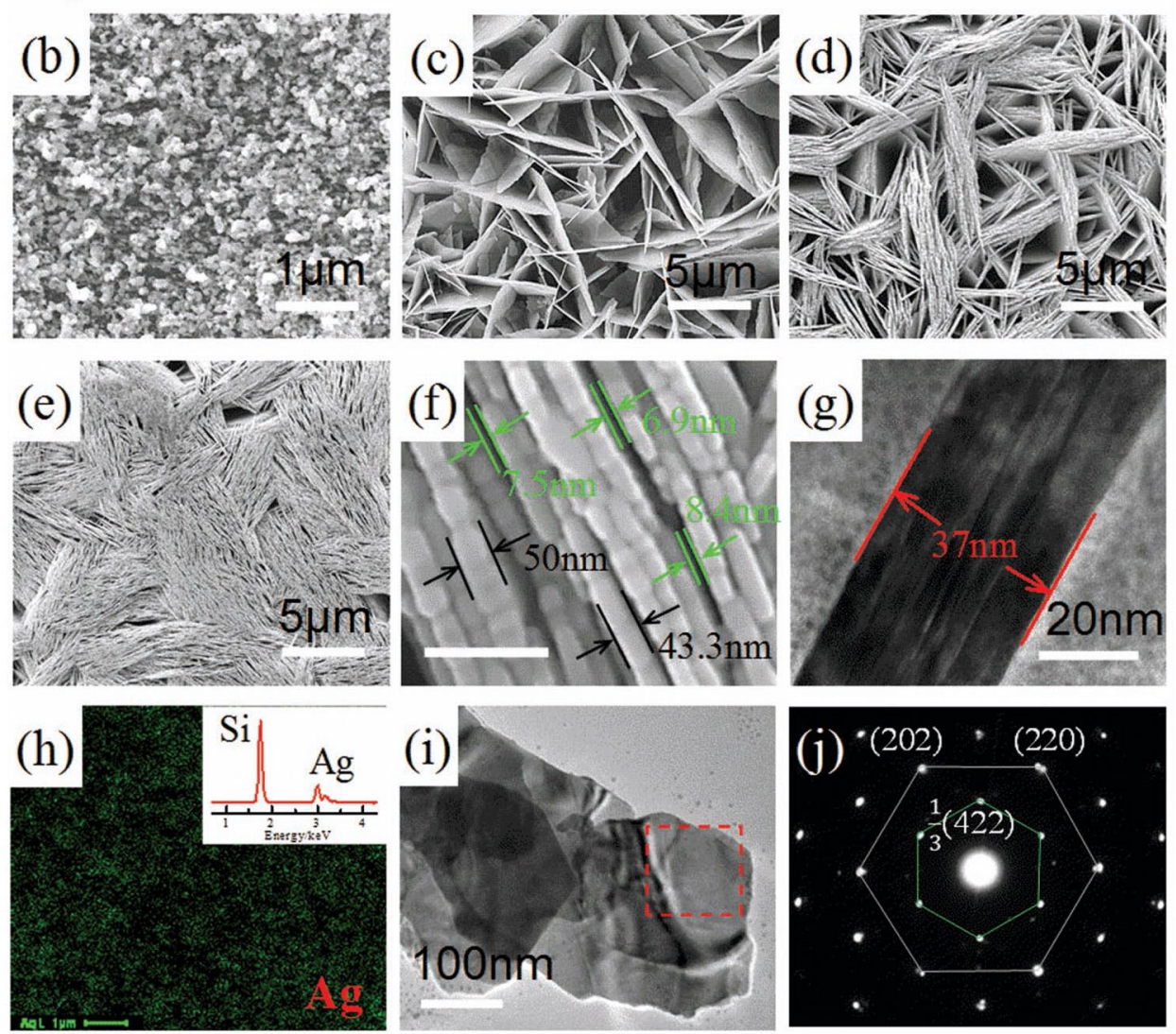

Figure 2. Growing process and characterizations of as-prepared AgNPs arrays. a) Schematic illustration of the anisotropic growth of AgNPs on silicon. b-e) Typical SEM images of silver nanostructures at different growing time of about $0,5,8$, and $10 \mathrm{~h}$, respectively. $\mathrm{f}$ ) The magnified picture of AgNPs. The scale bar is $200 \mathrm{~nm} . \mathrm{g}$ ) TEM image of cross-section of single AgNP. h) EDS mapping of silver element distribution in (b). The inset is the corresponding EDS spectrum. The scale bar is $1 \mu \mathrm{m}$. i) and j) are the TEM image of AgNPs and the corresponding SAED pattern.

(Figure S4, Supporting Information). Importantly, no silicon element is detected on AgNPs (Figure S5, Supporting Information), demonstrating the reduction of metal cations by hydrated electrons or radicals in plasma other than silicon atomic clusters. 
In order to figure out the growth mechanism of AgNPs, selected area electron diffraction (SAED) pattern is collected when the electron beam is vertically incident to the face of AgNPs. As show in Figure $2 \mathrm{j}$, the green hexagonal spot arrays can be assigned to the 1/3(422) planes, which should be attributed to the (111) stacking faults parallel to the (111) surface for AgNPs. The white hexagonal spot arrays can be attributed to (220) Bragg reflections, ${ }^{[21]}$ which demonstrate that the asprepared AgNPs are single-crystal with (111) orientation. Therefore, the formation process of nanoplates can be concluded in Figure $2 a$. Briefly, the reduced $\mathrm{Ag}^{0}$ atoms will nucleate on the (110) surface plane of Ag seeds due to the highest surface energy: (110) $0.953 \mathrm{eV}>(100)$ $0.653 \mathrm{eV}>(111) 0.553 \mathrm{eV} \cdot{ }^{[21 b, 22]}$ And the formed nuclei will preferentially grow with the terminal (111) planes, leading to formation of AgNPs. ${ }^{[21 b]}$ However, when the concentration of $\mathrm{AgNO}_{3}$ is high $(>0.3 \mathrm{~m})$, all the surface planes of silver seeds have the chances to adsorb the extra dissociative $\mathrm{Ag}^{0}$, which will lead to isotropic growth into blocks (Figure S6, Supporting Information).

\subsection{Ultrafast Plasma Enhancing Photoreduction of Metal Cations}

In order to figure out the electron donors for grafting the reduced silver nanoparticles onto silicon substrate, control experiments were conducted. 1) Silicon wafer was ablated in deionized water, and then immersed in $\mathrm{AgNO}_{3}$ solution. Arguably, the silicon-hydrogen (Si?H) bonds have the ability to reduce noble metallic ions. ${ }^{[23]}$ Laser ablation in liquid risks forming $\mathrm{Si}-\mathrm{H}$ bond on silicon surface to reduce the silver ions. But no distinguishable $\mathrm{Si}-\mathrm{H}$ signal from Fourier transform infrared spectroscopy (Figure S7, Supporting Information) and no silver nanostructures were observed. 2) Laser irradiation of $\mathrm{AgNO}_{3}$ solution when focusing at about $3 \mathrm{~mm}$ above the silicon-liquid interface. Also no metallic structures were obtained on silicon surface even after ten days growth, except for Ag nanoparticles $(<5 \mathrm{~nm})$ suspended in the irradiated solution (Figure S8, Supporting Information). Although silver nitrate can be photoreduced by direct irradiation, the absence of fixed growing sites failed to assemble Ag nanoparticles into nanoplates. Therefore, reduction of $\mathrm{Ag}^{+}$by plasma and trapping $\mathrm{Ag}^{0}$ onto structured silicon are the two prerequisites for fabricating such plasmonic nanostructures on the given substrates. 
(a)

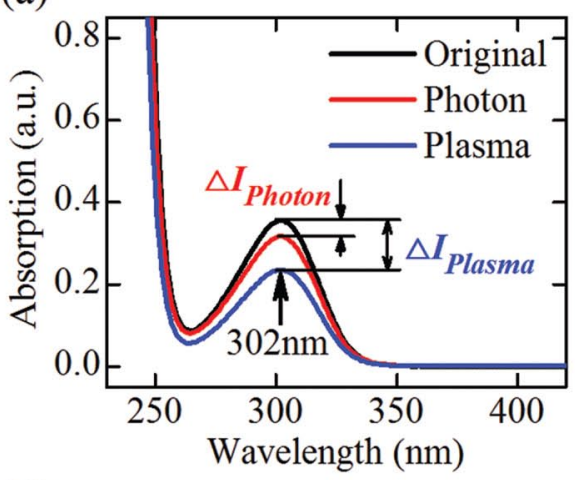

(d)

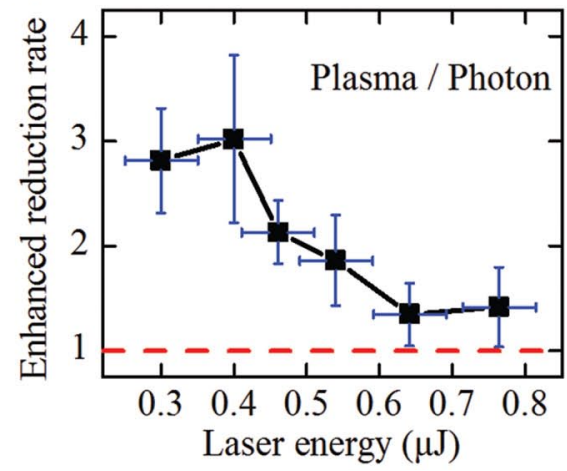

(b)

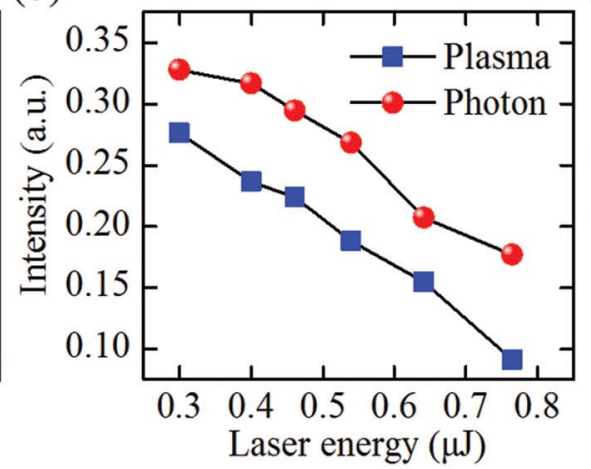

(e)

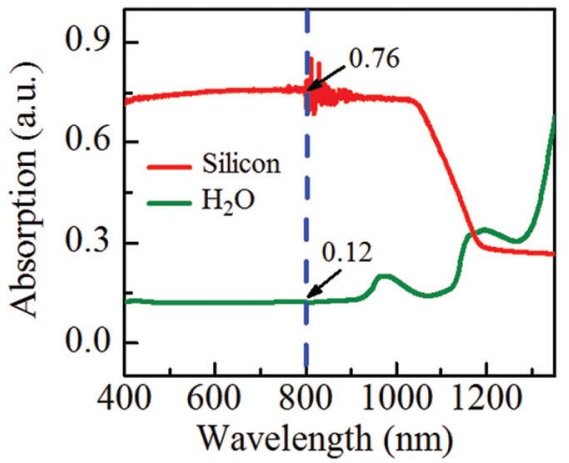

(c)

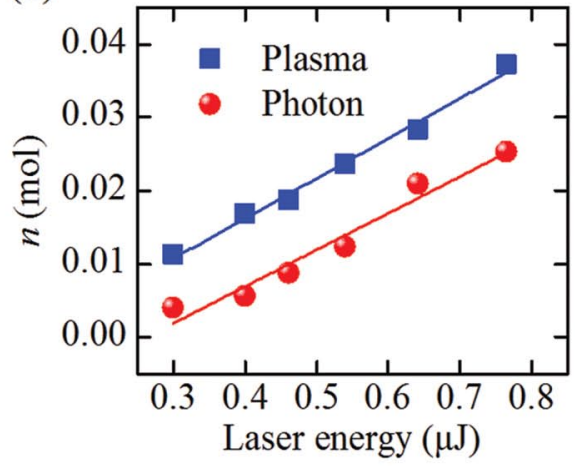

(f)

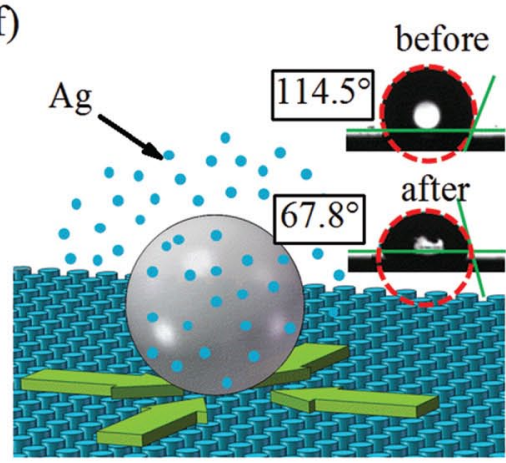

Figure 3. Laser-mediated-plasma enhanced reduction of metal cations. a) Comparison of absorption intensity changes between direct photon-reduction and plasmacatalysis reduction of silver nitrate at a fixed incident energy of $0.4 \mu \mathrm{J}$ (other form factors were the same). b) Intensities plotted as a function of laser energy before and after irradiation. c) The number of transferred electrons by estimating the change in the absorbance. d) The rate enhancement calculated by dividing plasma-induced electron transfer by that of photon-reduction process as a function of incident laser energy. e) Absorption spectroscopy of silicon and deionized water. f) Schematic diagram of $\mathrm{Ag}^{0}$ concentrating to the irradiated area driven by surface tension gradient.

The performances of plasma are further demonstrated detailedly compared with laser ablation in metallic salt solution. As shown in Figure 3a, spectrum represents trapped electrons in irradiated $\mathrm{AgNO}_{3}$ solution with a broad absorption peaked at $302 \mathrm{~nm} \cdot{ }^{[24]}$ Due to the reduction and nucleation of silver ions, the absorption intensities decreased after direct photoirradiation ("photon") or plasma-reduction ("plasma") process. A significant decrease in the absorption intensity caused by plasma effect was observed over direct photoirradiation, indicating a higher photon utilization efficiency and quantum yield. It has been established that the intensity of UV-vis spectrum is 
proportional to molar concentration of solutes. ${ }^{[24]}$ One can analyze the absorption intensity by using the traditional "analog" method to correlate the intensity of one to the known solute concentration, thereby developing an intensity versus concentration calibration curve (Figure S9, Supporting Information). Thus, we can estimate the number of moles of electrons, $n(e)$, transferred to $\mathrm{Ag}^{+}$,

$$
n(e)=N \times \Delta C=N \times \Delta l \times C_{\text {original }} / l_{\text {original }}
$$

by monitoring the decrease in the absorbance. $N$ is the number of electrons to reduce metal ions $\left(N=1\right.$ for $\left.\mathrm{Ag}^{+}\right), \Delta C$ and $\Delta /$ are the differences of concentration and absorption intensity before and after irradiation, respectively, $I_{\text {original }}=0.3559$ and $C_{\text {original }}=0.05 \mathrm{~mol} \mathrm{~L}^{-1}$ are the intensity and concentration of precursor solution $\left(\mathrm{AgNO}_{3}\right)$, respectively. The intensities and the corresponding intensity changes are experimentally plotted in Figure 3b and Figure S10 (Supporting Information), respectively. Accordingly, the extracted electrons for reduction of silver cations are quantitatively calculated, and both exhibit linear dependences on the incident laser (Figure 3c), indicating a photochemical reaction rather than a photothermal process. ${ }^{[25]}$ From the slope of the plot, we obtained a gain value of about 0.05 and 0.054 moles of electrons per milliwatt of laser energy for photon and plasma, respectively, indicating laser-mediated plasma an additional function in reduction of silver cations.

In addition, we calculated the ratios of electrons transfer in plasma reduction to that in photon following the formula: $\gamma(e)=n_{\text {plasma }} / n_{\text {photon }}$. As plotted in Figure $3 d$, laser-mediated plasma can promote the ratios up to three-times than direct irradiation, which attribute to reduction of silver ions by the high active species (electrons, ions) involved in the plasma plume. ${ }^{[9 b]}$ Whereas experiment results exceeding our predictions but reasonable that the reduction yields gradually drop to an identical level, which fundamentally attributed to the terminated differences in energy-dependent photoabsorption between water and silicon. In order to confirm the conjecture, the absorption spectrum of silicon and water are conducted. As shown in the Figure $3 \mathrm{e}$, the used deionized water is of high transparency (absorption $<20 \%$ ), when exciting wavelength locating at around $800 \mathrm{~nm}(1.55 \mathrm{eV})$. The raw silicon exhibits significant absorption (>70\%) due to the photon energy larger than the bandgap of silicon (1.12 eV). Photoabsorption by silicon dominates at low laser energy. That is, laser-mediated silicon plasma and silicon plasma-induced water plasma significantly 
contribute to the elevated efficiency for reduction of silver cations. When at a higher laser energy, the ionization of water by laser excitation will generate water plasma before energetic photons arriving at silicon surface, ${ }^{[26]}$ resulting in the comparable ability in reduction of silver ions. That is, the competition of exciting silicon plasma or water plasma determines the final structures, which further supports our viewpoints.

In a conventional photocatalysis process, photogenerated electronhole pairs will recombine in the bulk or on catalyst surface, or separate and then migrate to surface to participate in given redox reactions. ${ }^{[3]}$ Unlike such process, we conclude the proposed mechanism of plasma-catalysis reduction of metal cation as a "trapping" process, where reactions proceed during laser-induced microexplosion. Generally, the reduction of silver nitrate is accepted as a multiphoton process. ${ }^{[27]}$ Much more external energy is required to exceed the chemical barriers to trigger the reduction reaction. Actually, $\mathrm{Ag}^{+}$can be reduced to $\mathrm{Ag}^{0}$ by capturing an electron from neighboring donors. Active plasma, which carries a flood of high energetic species (electrons, atomic clusters), can lower the barriers (less external energy) and accelerate the reduction reaction proceeding. That is, the upstream electrons or ions involved in plasma plume are trapped by surrounding metal ions, which may open an avenue in plasma technology and other fields.

More importantly, we found that the laser-modified regions were more hydrophilic $\left(67.8^{\circ}\right)$ than the pristine surface $\left(114.5^{\circ}\right)$ due the formation of ripple structures, as shown in Figure $3 f$ and Figure $S 11$ (Supporting Information). Arguably, micro/ nanostructured surfaces exhibit distinct wettability to originals, which could change the microscopic hydrodynamics during self-assembled growth. ${ }^{[28]}$ In our condition, Ag precursors suspended in liquid gradually concentrate and attach on the seeded surface due to the gradient of surface tension, and the subsequent Marangoni flow build great feats to the dense AgNPs formation. ${ }^{[28]}$

As we know, reduction of $\mathrm{Ag}^{+}$to $\mathrm{Ag}^{0}$ is to capture single electron from surroundings. In order to exploit and validate the universality of the proposed conceptual mechanism to fabricate functional nanomaterials, a redox reaction involving a 3-electron transfer to $\mathrm{Au}^{3+}$ is conducted. Except for a replacement of $\mathrm{AgNO}_{3}$ by $\mathrm{HAuCl}_{4}$, other form factors were the same. As shown in Figure S12 (Supporting Information), 
closely arranged Au microspheres are distinguishable in the SEM images after irradiation. The characteristic lines of Au attribute to the successful reduction of $\mathrm{Au}^{3+}$, which strongly support our basic idea. This introduced intermediation (silicon plasma) as the extra electron donor offers benefits for single but also multielectron reduction processes.

\subsection{Finite-Difference Time-Domain (FDTD) Simulations of En- hanced Electromagnetic Field}

Generally, SERS hot spots are generated by morphology dependent electromagnetic (EM) field confined between the adjacent plasmonic components or high-curvature sites. ${ }^{[1 b, 4 b, 6 b, 8]}$ Therefore, the SERS sensitivity can be significantly improved by decorating the plasmonic components with abundant nanogaps and sharp corners. ${ }^{[4 a]}$ The selfassembly process of AgNPs driven by surface tension gradient is unconstrained, enabling the emergence of multifarious AgNPs. As schematically shown in Figure 4a, AgNPs graft on silicon surface at different angles and orientations. Bundles of parallel-aligned AgNPs are interlaced together in Figure $4 \mathrm{~b}$, and the combination of nanogaps and joints may endow huge potentials in SERS enhancements for biosensors. For studying the effect of AgNPs arrays on the enhancement and distribution of the electromagnetic field on aligned NPs, FDTD simulations were performed on coupled, isolated, and cross-linked AgNPs. As shown in Figure 4d, EM field enhancements are observed at the nanogaps between the adjacent AgNPs. The electromagnetic enhancements are critically dependent on the gaps of adjacent AgNPs. The maximum enhancement of EM field was estimated to be 144 when the gap decreased to $10 \mathrm{~nm}$, an enhancement of around 1.99 and 27.5 compared to the cases of 20 and $50 \mathrm{~nm}$, respectively. The simulation results also reveal that the enhanced electromagnetic field peaked to about 7.9 at the sharp corners (Figure 4e), and exceeding 5 in the cross (Figure 4f). Meanwhile, the enhanced field decreases rapidly away from the AgNPs-air interfaces. We calculated the maximum values locating at interfaces $(0 \mathrm{~nm})$, but smaller values at above $(10 \mathrm{~nm})$ and below $(-10 \mathrm{~nm})$ the interface (Figure $4 \mathrm{f})$. More detailed simulations demonstrated that the maximum enhancements also depend on the angles between laser polarizations and longitudinal orientation of AgNPs (Figure S13, Supporting Information). Accordingly, this huge enhanced field induced by LSPR may offer full opportunities 
(a)

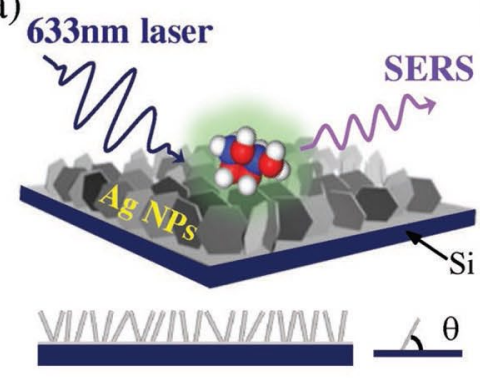

(d)

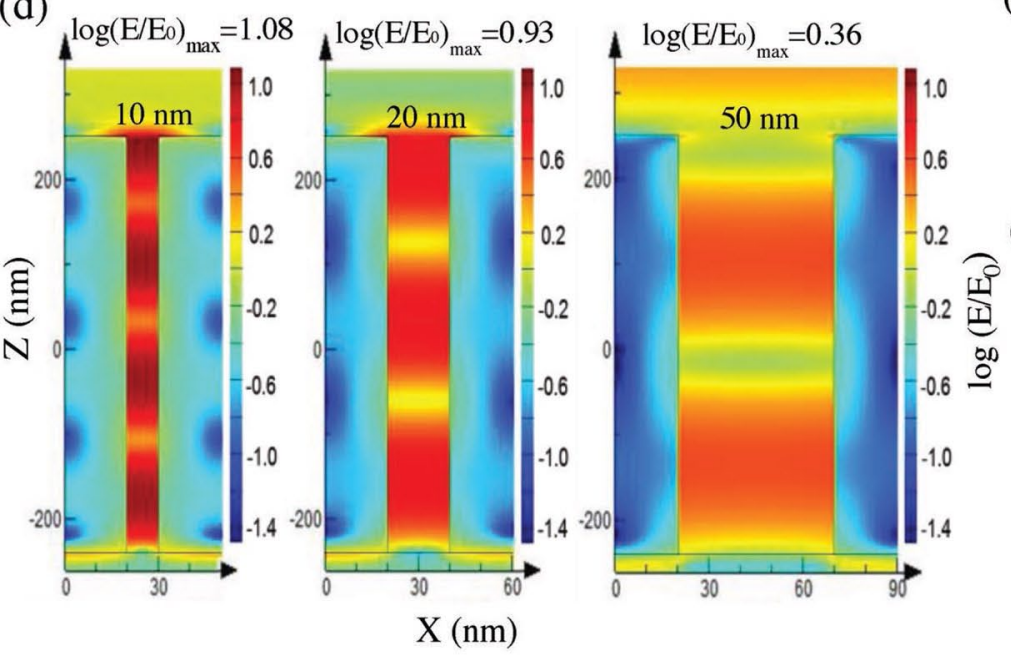

(b)

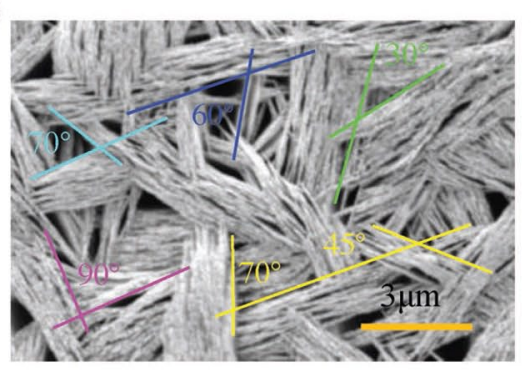

(c)

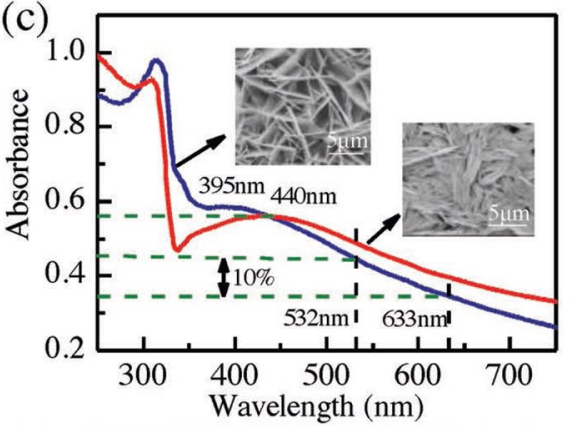

(e)
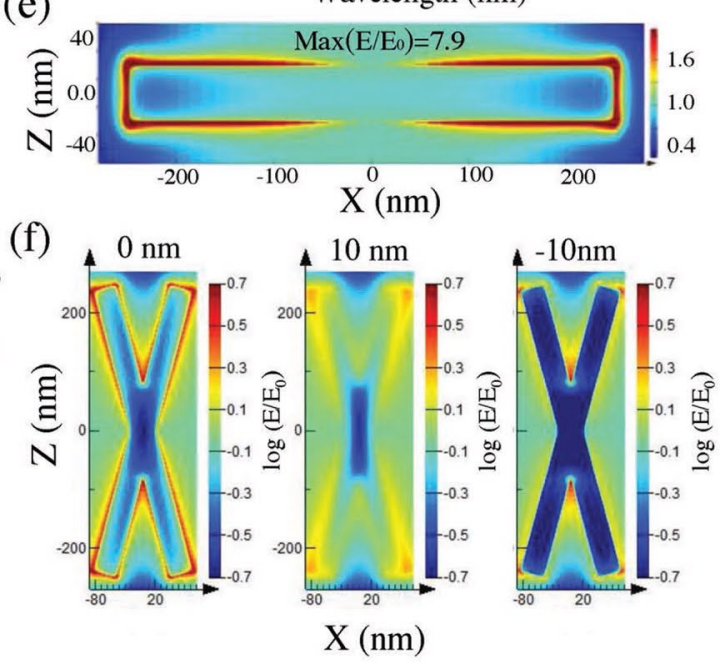

Figure 4. Plasmonic properties of aligned AgNPs. a) Schematic diagram and b) SEM image of AgNPs arrays. c) The UV-vis spectrum of the AgNPs arrays. d-f) FDTD simulations of electromagnetic enhancement of d) coupled, e) isolated, and f) $30^{\circ}$ crosslinked AgNPs. The incident laser wavelength in the simulations is $633 \mathrm{~nm}$.

to amplify the Raman scattering of biochemical molecules near metal nanostructures, even when trace analyte detection down to few-tosingle molecule level.

Furthermore, we carefully examine the optical properties of the substrates with different densities of AgNPs arrays. As shown in Figure 4c, the Ag nanoplates arrays possess strong absorption in the visible region, and the plasmon absorption band peaking at 395 and $440 \mathrm{~nm}$ can be observed. Notably, the surface plasmon absorption bands are significantly broadened across the whole visible region $(0.56$ for $440 \mathrm{~nm}$ and 0.347 for $633 \mathrm{~nm}$ ). Therefore, the Raman intensity of the detected molecules will be significantly enhanced when the frequencies of the impinged Raman lasers locate at the region of the broad absorption band of AgNPs arrays through exciting surface plasmon resonance (SPR). 


\subsection{High Sensitive and Dual-Analyte SERS Detections on Silver NPs Arrays}

In order to expose the superior plasmonic characters, the prepared AgNPs pairs are used for detecting R6G, MNBI, and 4-MBA. R6G is representative probe molecule in Raman detection experiments due to its high Raman scattering cross-section. The characteristic lines of R6G can be clearly identified in Figure $5 \mathrm{a}$, where 606 and $765 \mathrm{~cm}^{-1}$ peaks are assigned to $\mathrm{C}-\mathrm{C}-\mathrm{C}$ ring in-plane, out-of-plane bending, and $\mathrm{C}-\mathrm{H}$ in-plane bending vibrations, respectively, and the bands locating at $1122,1176,1355,1503,1567$, and $1643 \mathrm{~cm}^{-1}$ are associated with symmetric modes of in-plane $\mathrm{C}-\mathrm{C}$ stretching vibrations. ${ }^{[29]}$ To pursue the maximum sensitivity, we compared the SERS results of silver nanostructures with different growth time. As shown in Figure S14 (Supporting Information), the SERS intensities of R6G ( $\approx 1643$ $\mathrm{cm}^{-1}$ ) increase with the prolonging of growth time, which can attribute this enhancement to the increase of density of AgNPs and nanogaps between them, and the enhanced absorption of Raman laser (Figure 4c). Therefore, AgNPs array are employed to evaluate the SERS performances.

As shown in Figure $5 b$, the AgNP array exhibits high sensitivity toward R6G ranging from picomole $\left(\mathrm{p}_{\mathrm{m}}\right)$ to millimole $\left(\mathrm{m}_{\mathrm{m}}\right)$. The calibration curve ranging from $10^{-9}$ to $10^{-3} \mathrm{M}$ is relatively monotonic to annalyte concentration. Whereas this linearity is lost at higher concentrations $\left(>1 \times 10^{-3} \mathrm{M}\right)$ or lower ones $\left(<1 \times 10^{-9} \mathrm{M}\right)$. The limit of detection (LOD) was measured to be about $3 \times 10^{-12} \mathrm{M}\left(\approx 1.44 \mathrm{mg} \mathrm{m}^{-3}\right)$ for the AgNPs arrays. And the SERS substrate can keep high sensitivity when stored in water under dark environment for two months (Figure S15, Supporting Information). Meanwhile, to demonstrate the reproducibility of the AgNPs arrays, SERS mapping of R6G with a concentration of $3 \times 10^{-6} \mathrm{M}$ was recorded on a $80 \mu \mathrm{m} \times 80 \mu \mathrm{m}$ area (Figure 5c). The RSD of the peak intensities at $1643 \mathrm{~cm}^{-1}$ was calculated to be $5.3 \%$, confirming excellent uniformity of SERS signals. The limits of detection of R6G molecules have been compared with a commercial SERS chip (Figure S16, Supporting Information). The as-prepared AgNPs arrays exhibit better SERS performances of sensitivity (six times enhancement) and uniformity (RSD $=6.3 \%$ ) than the as-purchased commercial one (RSD $=21.7 \%)$. 

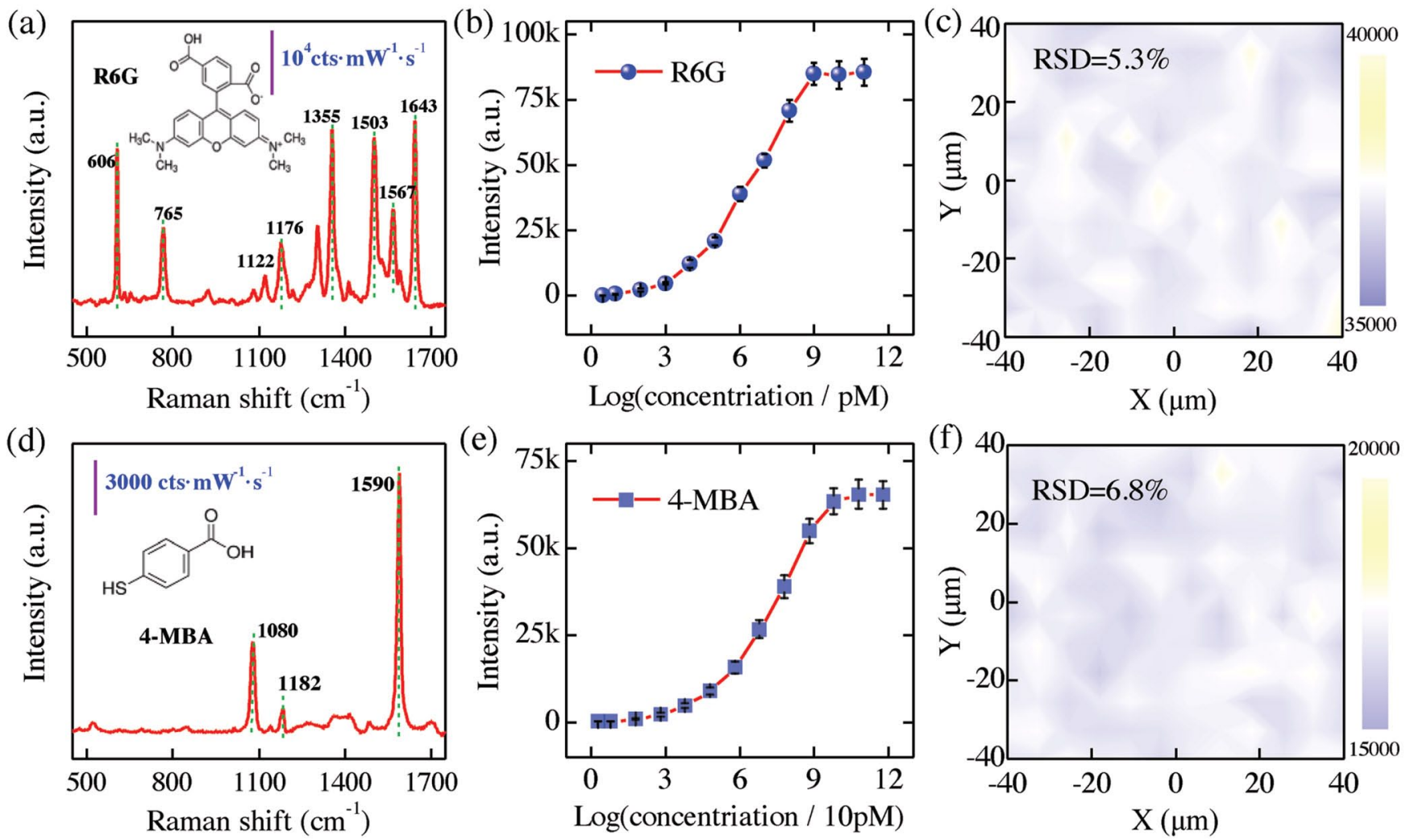

Figure 5. SERS detections of environmental pollutants with the AgNPs arrays. a) SERS spectrum of $3 \times 10^{-6} \mathrm{M}$ Rhodamine 6G (R6G) in aqueous solution. b) Peak intensities at $1643 \mathrm{~cm}^{-1}$ band plotted as the function of the logarithm of R6G concentration. c) SERS mapping of R6G $\left(1643 \mathrm{~cm}^{-1}\right)$ at a concentration of $3 \times 10^{-6} \mathrm{M}$ on randomly selected $80 \mu \mathrm{m} \times 80 \mu \mathrm{m}$ area. d) SERS spectrum of $6.5 \times 10^{-6} \mathrm{M} 4$-mercaptobenzoic acid (4-MBA) aqueous solution. e) Peak intensities at $1590 \mathrm{~cm}^{-1}$ band as the function of the logarithm of 4-MBA concentration. $f$ ) SERS mapping of 4-MBA $\left(1590 \mathrm{~cm}^{-1}\right)$ with a concentration of $6.5 \times 10^{-6} \mathrm{M}$ on randomly selected $80 \mu \mathrm{m} \times 80$ $\mu \mathrm{m}$ area.

Furthermore, the as-prepared SERS substrates were employed to detect the MNBI, a low Raman scattering crosssection analyte. As presented in Figure S17 (Supporting Information), a concentration down to $51 \times 10^{-12} \mathrm{M}$ has been successfully detected, displaying huge potentials in biochemical detections. Also an organic molecule of 4-MBA, which has strong chemical interactions with metal surfaces due to the existence of a thiol group and a carboxylic acid, is selected as the target analyte to study the SERS sensitivity of AgNPs. Seven characteristic peaks of 4-MBA are distinguished in the SERS spectrum, as shown 
in Figure $5 \mathrm{~d}$. Two peaks of 1590 and $1180 \mathrm{~cm}^{-1}$ dominated in the SERS spectrum are the $v^{8 a}$ and $v^{12}$ aromatic ring vibrations, respectively; while other peaks at $\approx 1150$ and $\approx 1180 \mathrm{~cm}^{-1}$ are assigned to the $\mathrm{C}-\mathrm{H}$ deformation vibrations. ${ }^{[30]}$ Also, the as prepared SERS substrates display wide detection range between tens picomole $\left(\mathrm{p}_{\mathrm{m}}\right)$ to millimole $\left(\mathrm{m}_{\mathrm{m}}\right)$ (Figure $5 \mathrm{e}$ ). And the monotonic relationship to annalyte concentration from $10^{-7}$ to $10^{-2} \mathrm{M}$ is analyzed. Meanwhile, the LOD on AgNPs was also established, and the detection concentration can be lowered to about $65 \times 10^{-12} \mathrm{M}$, demonstrating excellent low concentration detection ability. The corresponding RSD of the peak intensities at 1590 $\mathrm{cm}^{-1}$ was calculated to be $6.8 \%$ (Figure $5 \mathrm{f}$ ).

Generally, the intensity of Raman signals depends mainly on the inherent Raman scattering cross-section of molecules, optical absorption band of molecules and Raman substrate, and excitation wavelength of Raman laser. The maximum Raman signal can be obtained when the absorption frequencies among the detected molecules, Raman substrate, and Raman laser are overlapped or even in equivalence, due to the excitation of SPR effect. ${ }^{[1 b, 4 c]}$ As shown in Figure S18a (Supporting Information), the comparison of signal intensity of R6G molecules under 633 and $532 \mathrm{~nm}$ Raman excitation, respectively, has been presented. The limit of detection ability can be lowered down to $30 \times 10^{-15} \mathrm{M}$, which has improved by two orders of magnitude. This may be attributed to the larger absorption (10\%) of $532 \mathrm{~nm}$ photon than that for $633 \mathrm{~nm}$ by AgNPs arrays (Figure 4c), and resonant absorption of $532 \mathrm{~nm}$ photons by R6G molecules (Figure S18c, Supporting Information). And also, this improvement in signal intensity can also be observed in the case of 4-MBA (Figure S18b, Supporting Information), due to the enhanced absorption of $532 \mathrm{~nm}$ light (Figure 4c).

Actually, in a real environment, the contaminated samples may contain a mixture of pollutants that need to be identified, as signals from molecules with larger molar ratio can swamp these from lower one. ${ }^{[4 b, c, 31]}$ Therefore, the ordered AgNPs arrays were used to detect a mixture of $3 \times 10^{-9} \mathrm{M}$ Rhodamine $6 \mathrm{G}$ and $6.5 \times 10^{-9} \mathrm{M} 4$-mercaptobenzoic acid in ethanol. Spectra containing both Raman reporters with a signal-to-noise ratio exceeding 3:1 were counted as true positives. The dependence of the mole ratios on the SERS intensity of the two selected analytes (R6G and 4-MBA) is concluded in Figure 6a-c, 

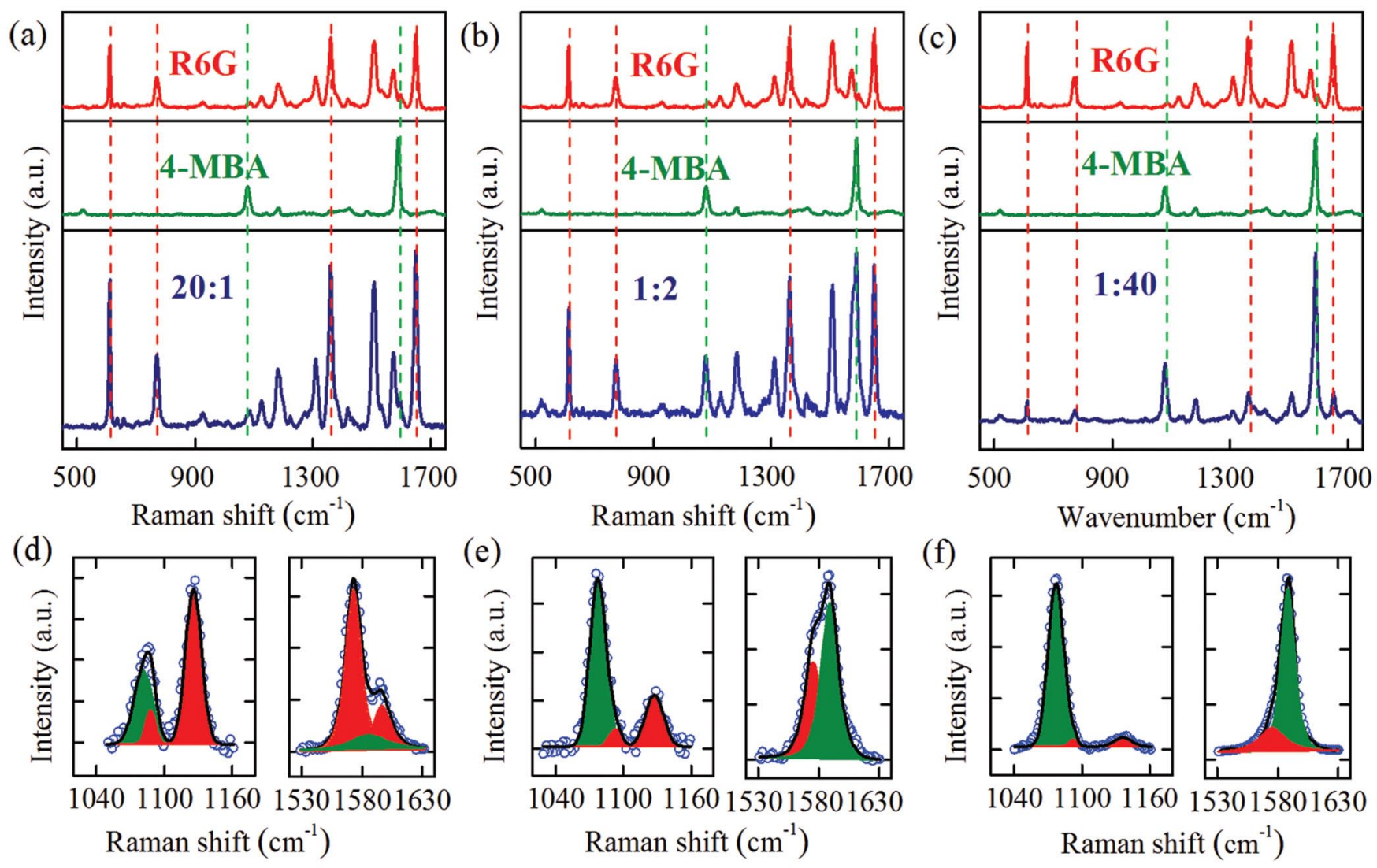

Figure 6. Dual-analyte detection of environmental pollutants using the AgNPs arrays for a mole ratio (R6G/4-MBA) of a) 20:1, b) 1:2, and c) 1:40. e,f) The amplified overlapped peaks correspond in (a), (b), and (c), respectively.

for mole ratios of 20:1, 1:2 and 1:40, respectively. Among all spectra, the characteristic lines of the both analytes could be observed and detected on each upper two panels. However, characteristic bands of 4-MBA (1590 and $\left.1080 \mathrm{~cm}^{-1}\right)$ are too close with these of R6G (1176 and $1567 \mathrm{~cm}^{-1}$ ) to be easily distinguished. We presume that the vibrational bands feature Gaussian profiles, so that we could figure out the intensity ratios between R6G and 4-MBA by peak fitting with Gaussian lines. As shown in Figure $6 \mathrm{~d}-\mathrm{f}$, the characteristic lines of R6G and 4-MBA can be analyzed by decomposing the neighboring or overlapped peaks with Gaussian mode. The good sensitivities of AgNPs arrays exhibit promising protential for trace multianalytes monitors, even featuring similar Raman lines. 


\section{Conclusions}

In summary, we propose a general strategy for in situ fabrication of metallic nanostructures via laser ablation at solid-liquid interface. The significance of laser-mediated plasma in the reduction of metal ions is highlighted. By trapping the upstream electrons during plasma by metal precursors, erupted reduction of metal cations into nanoparticles occurred through single/multielectron process, exhibiting higher photon utilization efficiency in formation of colloidal metal nanoparticles over conventional photoreduction process. Furthermore, favored by surface-tension gradient between laser irradiated and unirradiated regions, these photosynthetic metallic nanoparticles are accessible to selfassemble into nanoplates for silver and nanospheres for gold. These metallic nanostructures exhibit excellent SERS performances in trace detection of R6G, 4-MBA, and MNBI molecules with high sensitivity (down to $10^{-12} \mathrm{~mol} \mathrm{~L}^{-1}$ ) and reproducibility (RSD $<7 \%$ ), and good dual-analyte detection ability with mixture ratios of R6G to 4-MBA ranging from 20 to 0.025 . The synthetic role of plasma and customized surface-tension gradient in controllable preparation of metal nanostructures by direct laser writing method could provide exciting opportunities in photochemical reactions, plasmofluidic, and material synthesis.

\section{Experimental Section}

Fabrication of AgNPs: AgNO3 solution with concentration of 0.05 $\mathrm{m} \mathrm{L}^{-1}$ provided raw material for preparation of Ag nanostructures. $\mathrm{N}$ doped Si wafers were treated by an ethanol solution of FAS-17 (1.0 wt \%) for $5 \mathrm{~h}$, and then heated at $140^{\circ}$ for $1 \mathrm{~h}$. Direct-laser-writing method was conducted using a planoconvex lens $(f=100 \mathrm{~mm})$ setup equipped with a NIR fs laser (Specta Physics, Inc. 800 nm, 35 fs, 1 kHz) and a highprecision six-axis translation stage (M-840.5DG, PI, Inc.). The machining process was monitored by a CCD. For more detailed information, see Figure $\mathrm{S} 1$ of the Supporting Information. Therein precise control of focusing positions at silicon-liquid interface and processing parameters (scan speed and laser energy) are important for fabrication of these structures. The postirradiated samples experience a free growth process with controlled time to obtain AgNPs. 
Characterization of AgNPs: The surface morphology and EDS were conducted by a SEM (FEI Quanta 200 FEG). Transmission electron microscopy (TEM) images were obtained on a JEM-2100. The ultraviolet-visible (UV-vis) spectra were measured using an Agilent Cary-5000 spectrophotometer. The wettability of the samples was investigated with an optical angle-measuring device (OCA 15 Plus, Data Physics Instruments).

SERS Performances of AgNPs: Raman spectra were collected by a Renishaw InVia Reflex spectrometer with a $633 \mathrm{~nm}$ light source. For typical Raman tests, SERS chips dip in the analyte solutions to ensure the adsorption-desorption equilibrium between analyte (MNBI, R6G, and 4-MBA) and plasmonic nanostructures before experiments.

Supporting Information follows the References.

Acknowledgments - The research was supported by the National Key R\&D Program of China (Grant No. 2017YFB1104300) and National Natural Science Foundation of China (NSFC) (Grant No. 51775047).

Conflicts of Interest - The authors declare no conflict of interest.

\section{References}

[1] a) D. Punj, M. Mivelle, S. B. Moparthi, T. S. Van Zanten, H. Rigneault, N. F. Van Hulst, M. F. García-Parajó, J. Wenger, Nat. Nanotechnol. 2013, 8, 512; b) C. Zong, M. Xu, L.-J. Xu, T. Wei, X. Ma, X.-S. Zheng, R. Hu, B. Ren, Chem. Rev. 2018, 118, 4946; c) L. Yang, P. Li, H. Liu, X. Tang, J. Liu, Chem. Soc. Rev. 2015, 44, 2837; d) I. Amenabar, S. Poly, W. Nuansing, E. H. Hubrich, A. A. Govyadinov, F. Huth, R. Krutokhvostov, L. Zhang, M. Knez, J. Heberle, Nat. Commun. 2013, 4, 2890; e) Z. Yin, Y. Wang, C. Song, L. Zheng, N. Ma, X. Liu, S. Li, L. Lin, M. Li, Y. Xu, W. Li, G. Hu, Z. Fang, D. Ma, J. Am. Chem. Soc. 2018, $140,864$.

[2] a) Q. Dai, M. Ouyang, W. Yuan, J. Li, B. Guo, S. Lan, S. Liu, Q. Zhang, G. Lu, S. Tie, Adv. Mater. 2017, 29, 1701918; b) P. Zijlstra, J. W. Chon, M. Gu, Nature 2009, 459, 410.

[3] a) E. Cortés, W. Xie, J. Cambiasso, A. S. Jermyn, R. Sundararaman, P. Narang, S. Schlücker, S. A. Maier, Nat. Commun. 2017, 8, 14880; b) S. Linic, U. Aslam, C. Boerigter, M. Morabito, Nat. Mater. 2015, 14, 567; c) S. Linic, P. Christopher, D. B. Ingram, Nat. Mater. 2011, 10, 911; d) Y. Zhang, S. He, W. Guo, Y. Hu, J. Huang, J. R. Mulcahy, W. D. Wei, Chem. Rev. 2018, 118, 2927.

[4] a) S.-Y. Ding, J. Yi, J.-F. Li, B. Ren, D.-Y. Wu, R. Panneerselvam, Z.-Q. Tian, Nat. Rev. Mater. 2016, 1, 16021; b) W. Yan, L. Yang, J. Chen, Y. Wu, P. Wang, Z. Li, 
Adv. Mater. 2017, 29, 1702893; c) M. P. Cecchini, V. A. Turek, J. Paget, A. A. Kornyshev, J. B. Edel, Nat. Mater. 2013, 12, 165; d) J. W. Jeong, M. M. P. Arnob, K. M. Baek, S. Y. Lee, W. C. Shih, Y. S. Jung, Adv. Mater. 2016, 28, 8695; e) W. Lee, S. Y. Lee, R. M. Briber, O. Rabin, Adv. Funct. Mater. 2011, 21, 3424; f) J. E. George, V. K. Unnikrishnan, D. Mathur, S. Chidangil, S. D. George, Sens. Actuators, B 2018, 272, 485.

[5] a) T. Chen, B. Dong, K. Chen, F. Zhao, X. Cheng, C. Ma, S. Lee, P. Zhang, S. H. Kang, J. W. Ha, Chem. Rev. 2017, 117, 7510; b) J.-H. Zhong, X. Jin, L. Meng, X. Wang, H.-S. Su, Z.-L. Yang, C. T. Williams, B. Ren, Nat. Nanotechnol. 2017, 12, 132.

[6] a) S. Zhang, R. Geryak, J. Geldmeier, S. Kim, V. V. Tsukruk, Chem. Rev. 2017, 117, 12942; b) L. Scarabelli, M. Coronado-Puchau, J. J. Giner-Casares, J. Langer, L. M. Liz-MarzáN, ACS Nano 2014, 8, 5833; c) Z. Yin, W. Zhang, Q. Fu, H. Yue, W. Wei, P. Tang, W. Li, W. Li, L. Lin, G. Ma, D. Ma, Small 2014, 10, 3619.

[7] a) M. Grzelczak, L. M. Liz-Marzán, Chem. Soc. Rev. 2014, 43, 2089; b) M. R. Langille, M. L. Personick, C. A. Mirkin, Angew. Chem., Int. Ed. 2013, 52, 13910; c) R. Jin, Y. Cao, C. A. Mirkin, K. Kelly, G. C. Schatz, J. Zheng, Science 2001, 294, 1901; d) Y. Y. Cao, N. Takeyasu, T. Tanaka, X. M. Duan, S. Kawata, Small 2009, 5, 1144; e) C.-H. Lin, L. Jiang, Y.-H. Chai, H. Xiao, S.-J. Chen, H.-L. Tsai, Opt. Express 2009, 17, 21581.

[8] M. Chirumamilla, A. Toma, A. Gopalakrishnan, G. Das, R. P. Zaccaria, R. Krahne, E. Rondanina, M. Leoncini, C. Liberale, F. De Angelis, Adv. Mater. 2014, 26, 2353.

[9] a) J. Xiao, P. Liu, C. Wang, G. Yang, Prog. Mater. Sci. 2017, 87, 140; b) D. Zhang, B. GöKce, S. Barcikowski, Chem. Rev. 2017, 117, 3990; c) V. Amendola, M. Meneghetti, Phys. Chem. Chem. Phys. 2013, 15, 3027; d) H. Zeng, X. W. Du, S. C. Singh, S. A. Kulinich, S. Yang, J. He, W. Cai, Adv. Funct. Mater. 2012, 22, 1333; e) G. Yang, Prog. Mater. Sci. 2007, 52, 648; f) B. Pan, J. Xiao, J. Li, P. Liu, C. Wang, G. Yang, Sci. Adv. 2015, 1, e1500857; g) J. Xiao, J. Li, G. Yang, Small 2017, 13, 1603495.

[10] K. Y. Niu, L. Fang, R. Ye, D. Nordlund, M. M. Doeff, F. Lin, H. Zheng, Angew. Chem., Int. Ed. 2016, 55, 14272.

[11] Y. Yonezawa, T. Sato, M. Ohno, H. Hada, J. Chem. Soc., Faraday Trans. 1 1987, 83, 1559.

[12] a) R. C. Luo, C. Li, X. W. Du, J. Yang, Angew. Chem., Int. Ed. 2015, 54, 4787; b) J. Yang, T. Ling, W.-T. Wu, H. Liu, M.-R. Gao, C. Ling, L. Li, X.-W. Du, Nat. Commun. 2013, 4, 1695.

[13] a) F. Lin, J. Yang, S.-H. Lu, K.-Y. Niu, Y. Liu, J. Sun, X.-W. Du, J. Mater. Chem. 2010, 20, 1103; b) K. Niu, J. Yang, S. Kulinich, J. Sun, H. Li, X. Du, J. Am. Chem. Soc. 2010, 132, 9814.

[14] P. Li, Y. Li, Z. K. Zhou, S. Tang, X. F. Yu, S. Xiao, Z. Wu, Q. Xiao, Y. Zhao, H. Wang, Adv. Mater. 2016, 28, 2511.

[15] a) B.-B. Xu, Z.-C. Ma, L. Wang, R. Zhang, L.-G. Niu, Z. Yang, Y.-L. Zhang, W.-H. Zheng, B. Zhao, Y. Xu, Lab Chip 2011, 11, 3347; b) X. M. Li, M. H. Bi, L. Cui, Y. Z. Zhou, X. W. Du, S. Z. Qiao, J. Yang, Adv. Funct. Mater. 2017, 27, 1605703; c) 
A. Pallaoro, M. R. Hoonejani, G. B. Braun, C. D. Meinhart, M. Moskovits, ACS Nano 2015, 9, 4328; d) M. R. Lee, H. K. Lee, Y. Yang, C. S. L. Koh, C. L. Lay, Y. H. Lee, I. Y. Phang, X. Y. Ling, ACS Appl. Mater. Interfaces 2017, 9, 39584; e) W. Xie, R. Grzeschik, S. Schlücker, Angew. Chem., Int. Ed. 2016, 55, 13729.

[16] L. Jiang, A.-D. Wang, B. Li, T.-H. Cui, Y.-F. Lu, Light: Sci. Appl. 2018, 7, 17134.

[17] K. L. Choo, Y. Ogawa, G. Kanbargi, V. Otra, L. M. Raff, R. Komanduri, Mater. Sci. Eng. A 2004, 372, 145.

[18] a) C. Zhu, G. Meng, P. Zheng, Q. Huang, Z. Li, X. Hu, X. Wang, Z. Huang, F. Li, N. Wu, Adv. Mater. 2016, 28, 4871; b) Z. Zhang, Y. Fu, W. Yu, X. Qin, Z. Xue, Y. Liu, D. Luo, C. Yan, X. Sun, T. Wang, Adv. Mater. 2016, 28, 9589.

[19] a) Q. Wu, P. Diao, J. Sun, T. Jin, D. Xu, M. Xiang, J. Phys. Chem. C 2015, 119, 20709; b) Z. Li, G. Meng, T. Liang, Z. Zhang, X. Zhu, Appl. Surf. Sci. 2013, 264, 383.

[20] Y. Zhang, S. He, W. Guo, Y. Hu, J. Huang, J. R. Mulcahy, W. D. Wei, Chem. Rev. 2018, 118, 2927.

[21] a) G. Liu, W. Cai, L. Kong, G. Duan, F. Lü, J. Mater. Chem. 2010, 20, 767; b) Y. Xia, Y. Wu, T. Hang, J. Chang, M. Li, Langmuir 2016, 32, 3385.

[22] L. Polavarapu, L. M. Liz-Marzán, Phys. Chem. Chem. Phys. 2013, 15, 5288.

[23] Q. Shao, R. Que, M. Shao, L. Cheng, S. T. Lee, Adv. Funct. Mater. 2012, 22, 2067.

[24] M. Maillard, P. Huang, L. Brus, Nano Lett. 2003, 3, 1611.

[25] Y. Zhai, J. S. Duchene, Y.-C. Wang, J. Qiu, A. C. Johnston-Peck, B. You, W. Guo, B. Diciaccio, K. Qian, E. W. Zhao, Nat. Mater. 2016, 15, 889.

[26] a) J. Noack, A. Vogel, IEEE J. Quantum Electron. 1999, 35, 1156; b) A. Woutersen, U. Emmerichs, H. Bakker, Science 1997, 278, 658.

[27] B.-B. Xu, L. Wang, Z.-C. Ma, R. Zhang, Q.-D. Chen, C. Lv, B. Han, X.-Z. Xiao, X.L. Zhang, Y.-L. Zhang, ACS Nano 2014, 8, 6682.

[28] a) L. Bai, V. C. Mai, Y. Lim, S. Hou, H. Möhwald, H. Duan, Adv. Mater. 2018, 30, 1705667; b) J. Sun, B. Bao, M. He, H. Zhou, Y. Song, ACS Appl. Mater. Interfaces 2015, 7, 28086.

[29] H. Tang, G. Meng, Q. Huang, Z. Zhang, Z. Huang, C. Zhu, Adv. Funct. Mater. 2012, 22, 218.

[30] A. Michota, J. Bukowska, J. Raman Spectrosc. 2003, 34, 21.

[31] F. Sun, H.-C. Hung, A. Sinclair, P. Zhang, T. Bai, D. D. Galvan, P. Jain, B. Li, S. Jiang, Q. Yu, Nat. Commun. 2016, 7, 13437. 


\section{Supporting Information}

\section{Femtosecond photon-mediated plasma enhances photosynthesis of plasmonic nanostructures and their SERS applications}

Peng Ran, Lan Jiang, Xin Li, * Bo Li, Pei Zuo, and Yongfeng Lu

\section{Supplementary figures}
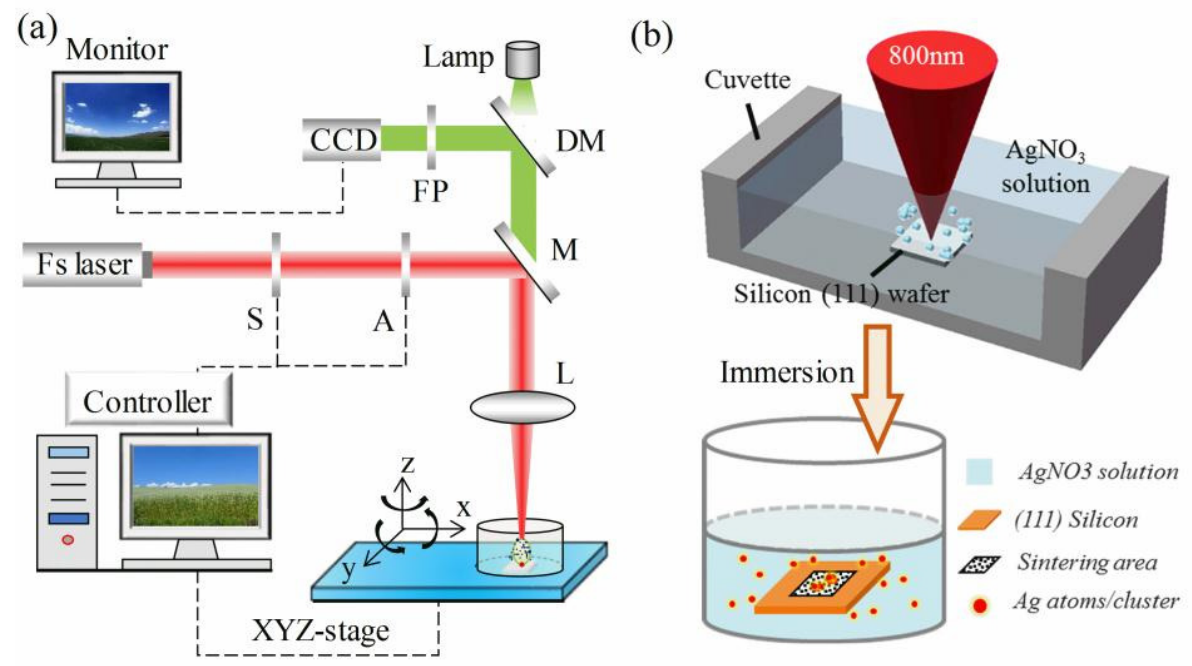

Figure S1 (a) Optical setup of the FsLDW. S is the shutter, $\mathrm{A}$ is the attenuator to control the laser power, FP is the filter plate, DM is the dichroic mirror, $\mathrm{M}$ is the ultrafast mirror, and $\mathrm{L}$ is the plano-convex lens ( $\mathrm{f}=100 \mathrm{~mm}$ ). (b) Self-assembly of Ag species into Ag NPs when stored in the dark.
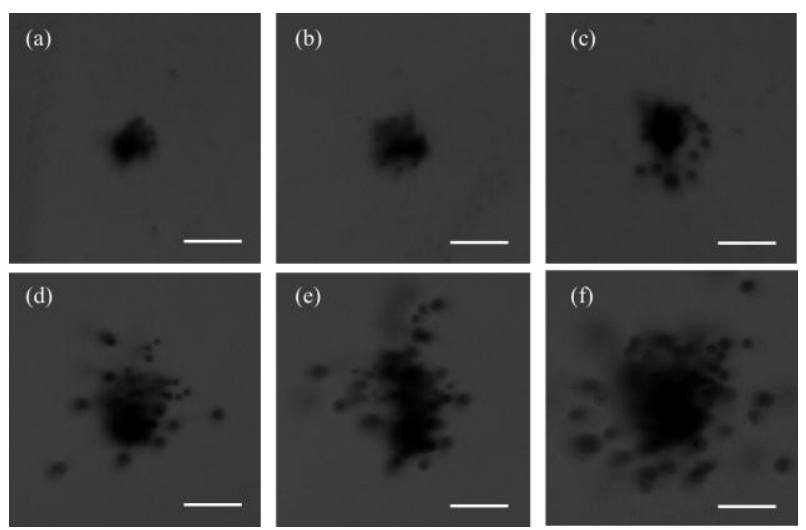

Figure S2 Pictures of plasma plume erupted from laser spots captured by a commercial CCD at different laser power of (a) $0.3 \mathrm{~mW}$, (b) $0.4 \mathrm{~mW}$, (c) $0.46 \mathrm{~mW}$, (d) $0.54 \mathrm{~mW}$, (e) $0.64 \mathrm{~mW}$, and (f) $0.76 \mathrm{~mW}$. The pictures are captured after one second when laser pulses terminated. The scale bars are $200 \mu \mathrm{m}$. 


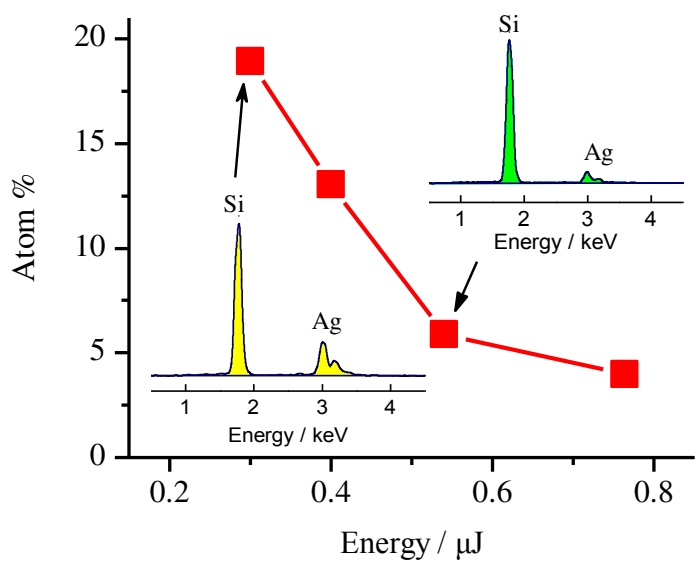

Figure S3 The energy dependence of the amount of Ag seeds sintered onto structured Si substrate. The insets are the corresponding EDS spectrum.
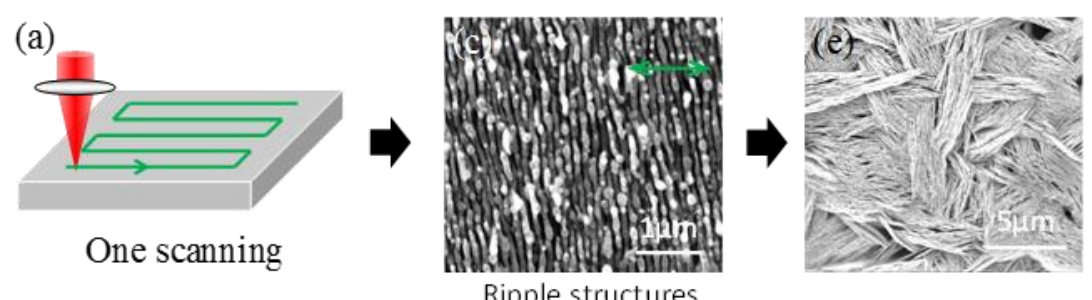

(b)
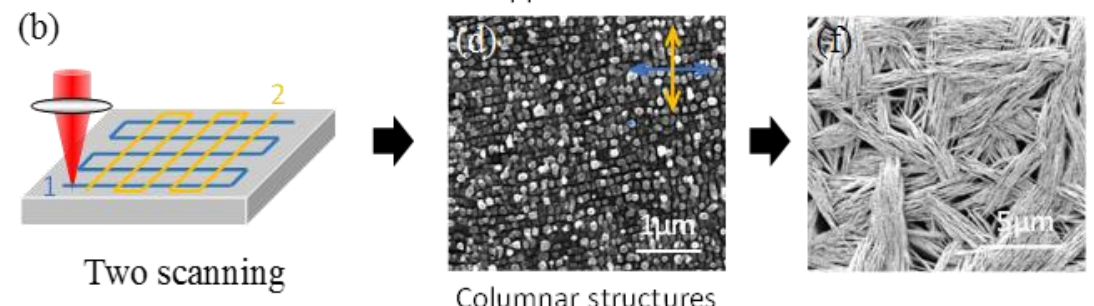

Columnar structures

Figure S4 (a) and (b) are the processing methods for the fabrication of ripple and columnar structures. (c) and (d) are the corresponding SEM images. (e) and (f) are the AgNPs arrays after growth time of 10 hours on ripple and columnar structures, respectively.

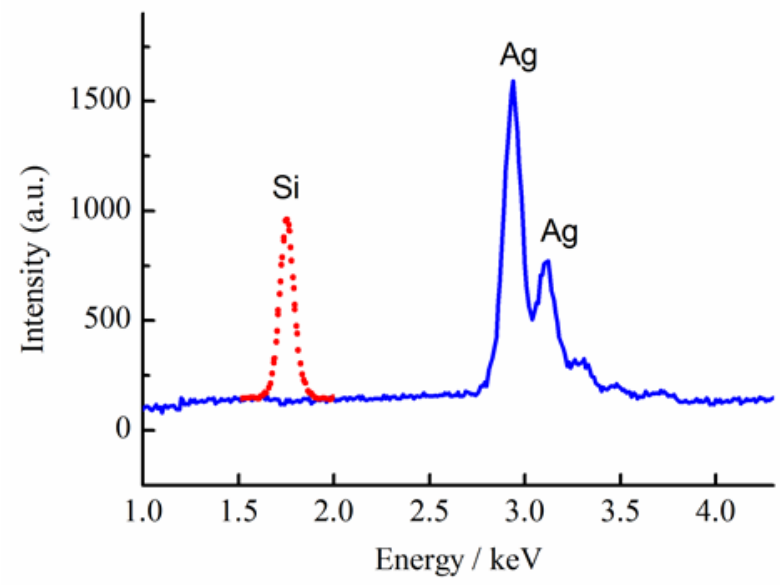

Figure S5 Energy dispersive X-ray spectroscopy (EDS) of dense AgNPs. No silicon element is detected (the signal of silicon is marked by red dashed line). 


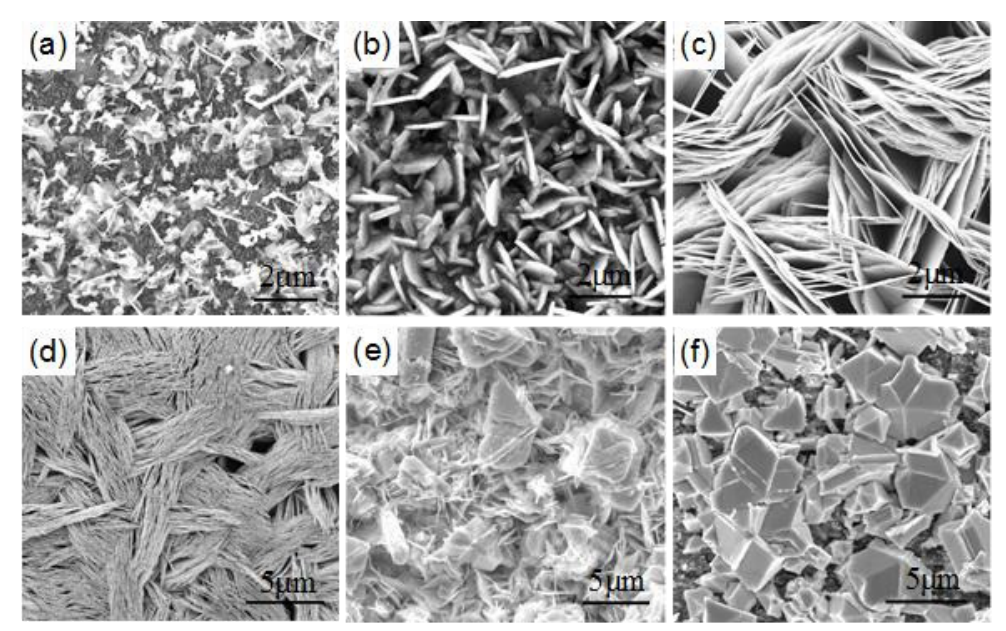

Figure S6 The influences of morphologies of silver nanostructures on $\mathrm{AgNO}_{3}$ concentration: (a) $0.01 \mathrm{M}$, (b) $0.03 \mathrm{M}$, (c) $0.05 \mathrm{M}$, (d) $0.1 \mathrm{M}$, (e) $0.3 \mathrm{M}$, and (f) $0.5 \mathrm{M}$.
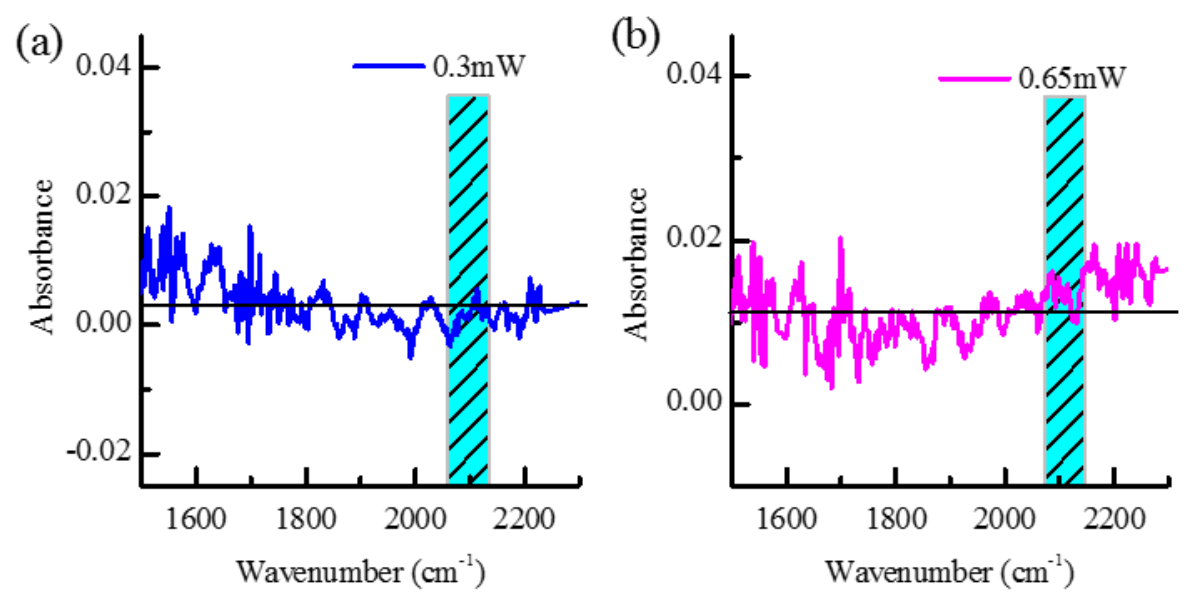

Figure S7 Fourier transform infrared (FTIR) spectrum of post-irradiated silicon at laser energy of (a) $0.3 \mathrm{~mW}$ and (b) $0.65 \mathrm{~mW}$. Other processing parameters were the same. No distinguishable signals of Si-H bonding $\left(\sim 2100 \mathrm{~cm}^{-1}\right)$ are presented.

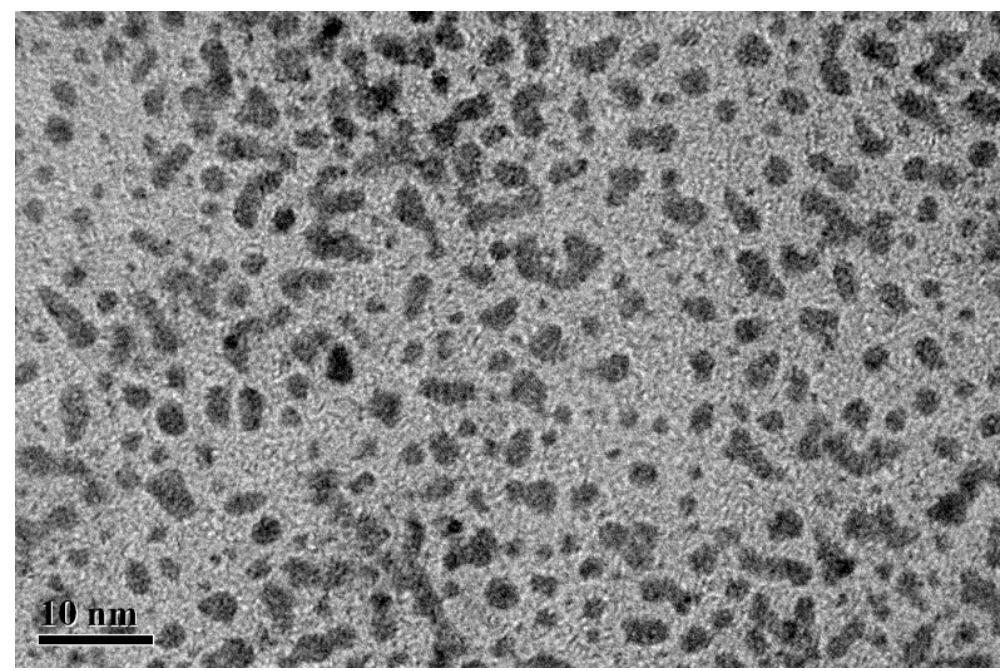

Figure S8 TEM of reduced Ag nanoparticles by direct $800 \mathrm{~nm}$ pulsed laser irradiation in 0.05 $\mathrm{mol} / \mathrm{L}$. 
Calibrating $\mathrm{AgNO}_{3}$ concentration as a function of absorption intensity. The UV-vis absorption measurements were performed via Varian Cary 5000 UV-Vis-NIR spectrophotometer. The samples were placed in optically transparent $1 \mathrm{~cm} \times 1 \mathrm{~cm}$ quartz cells. The quartz cells were carefully cleaned alternately using acetone, ethanol and distilled water in order to guarantee the reliability of the obtained data. Five independent measurements are averaged per data. We calibrated precisely the silver concentration as a function of absorption intensity: $\partial[\mathrm{Ag}+] / \partial \mathrm{I}=\mathrm{K}=0.131 \mathrm{~mol} \cdot \mathrm{L}^{-1}$, which corresponds to a linear behavior.
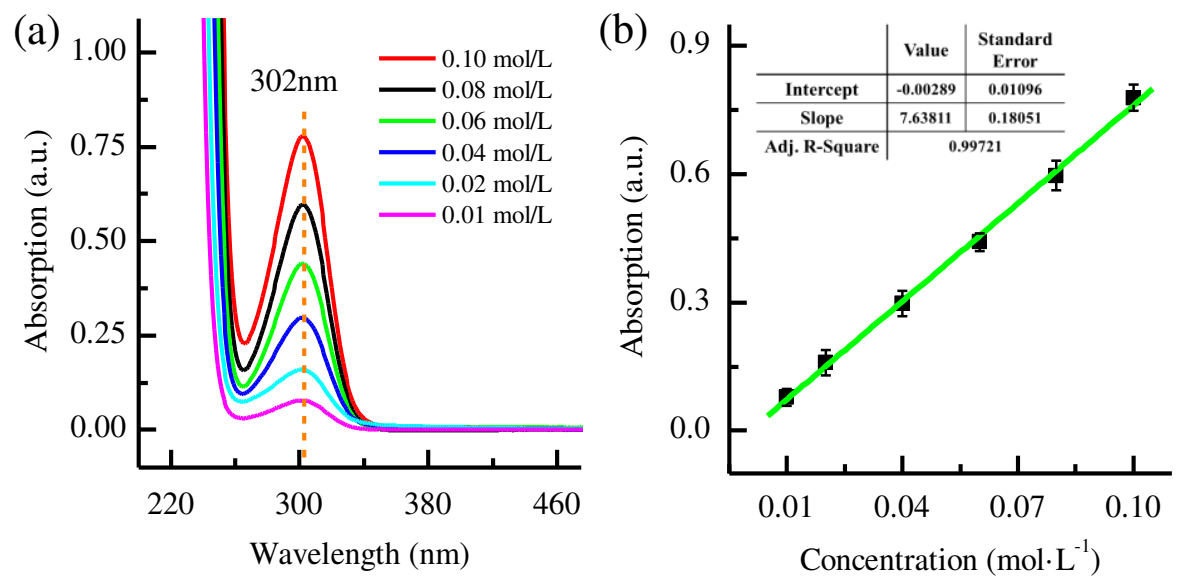

Figure S9 (a) Absorption spectrum of different concentration of silver nitrate $\left(\mathrm{AgNO}_{3}\right)$ from 0.01 to $0.1 \mathrm{~mol} / \mathrm{L}$. (b) Absorption intensities are plotted as a function of $\mathrm{AgNO}_{3}$ concentrations, revealing a perfect linear relationship. Each data averages the values of 5 independent measurements.

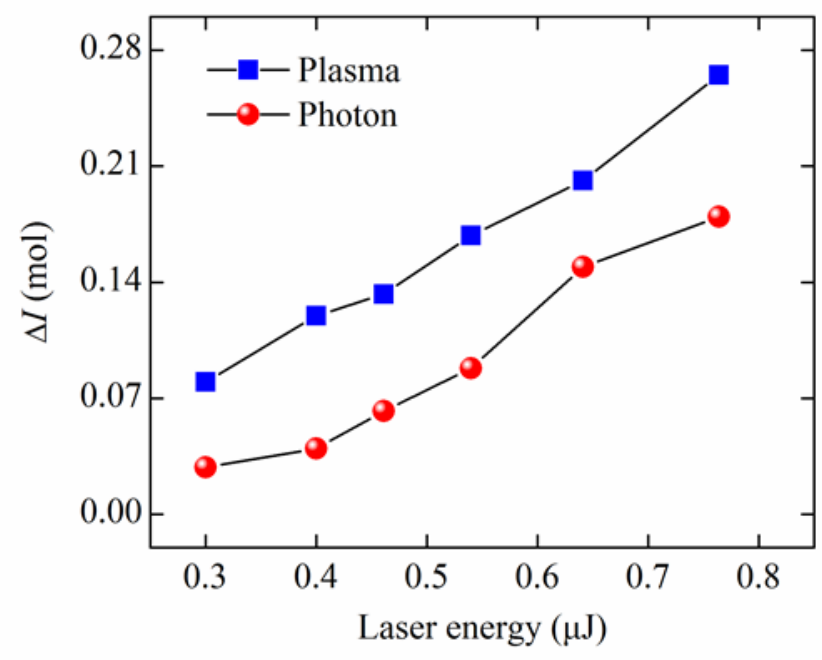

Figure S10 Changes of intensities plotted as a function of laser energy. 


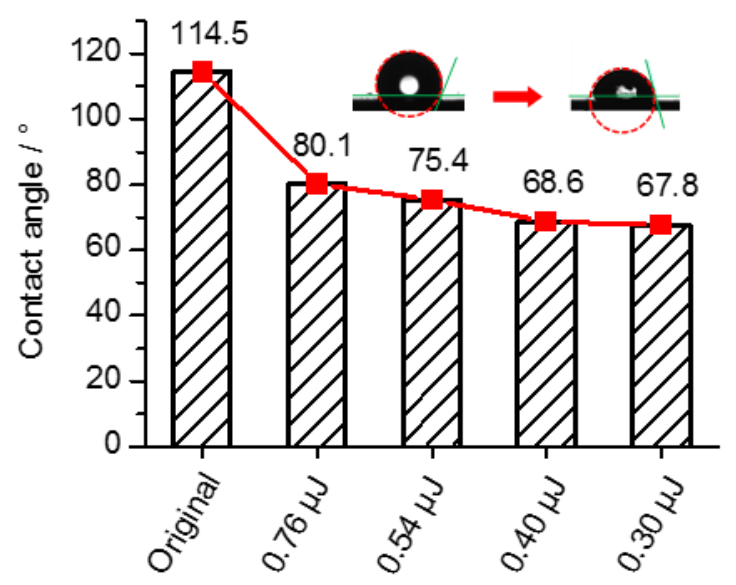

Figure S11 Contact angles change with the incident laser energies.
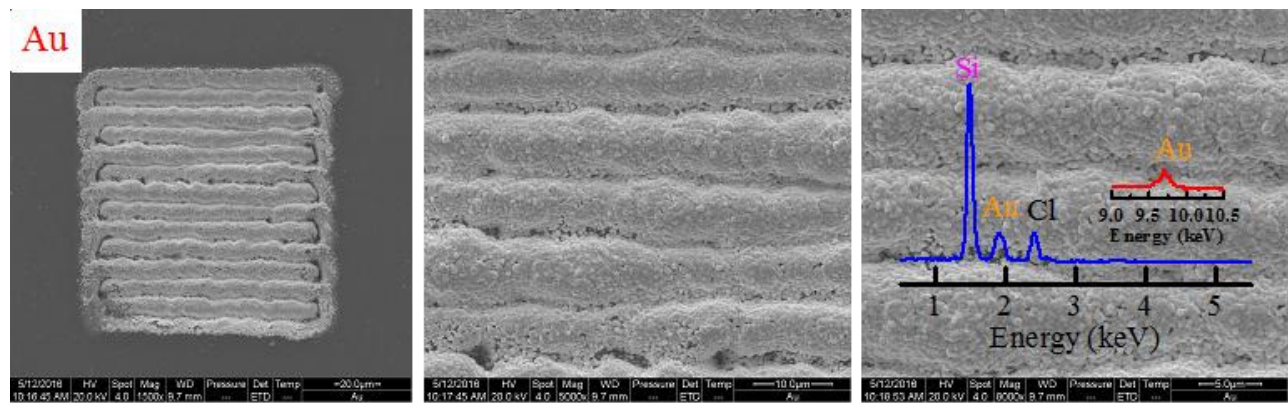

Figure S12 Fabrication of Au nanospheres on silicon by laser-mediated plasma. Laser power is $0.5 \mathrm{~mW}$, scanning speed is $100 \mu \mathrm{m} / \mathrm{s}$, and the growth time fixing at 10 hours. Other forms factors are same with the case of Ag. 


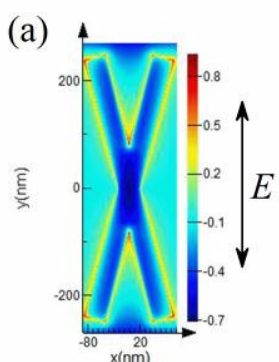

(b)

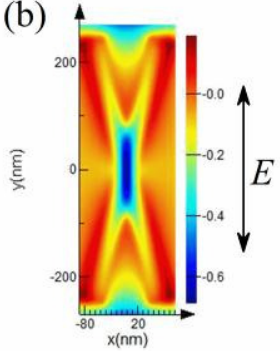

(c)

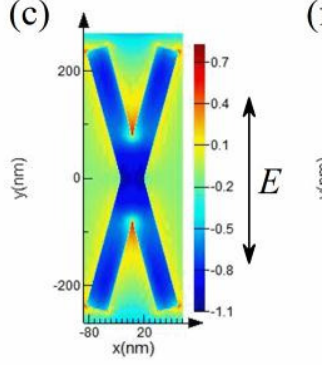

(d)

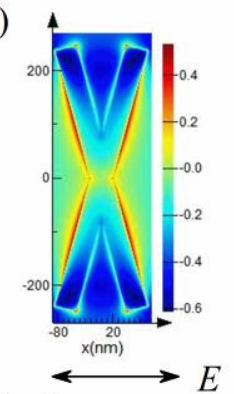

(e)

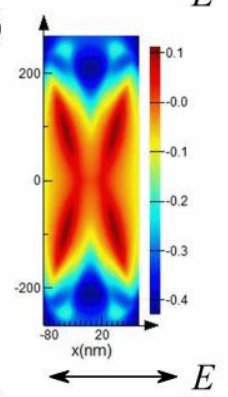

(f)

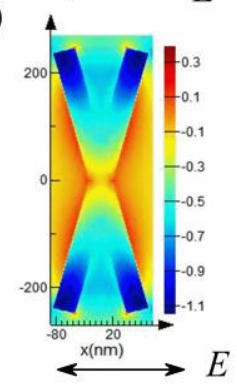

(g)

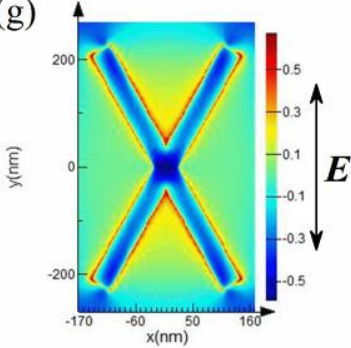

(h)

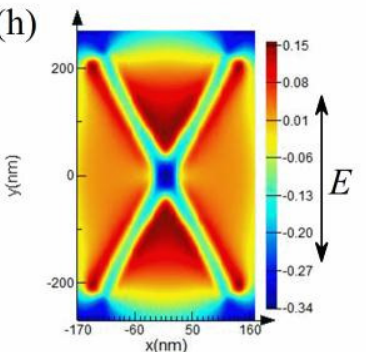

(i)

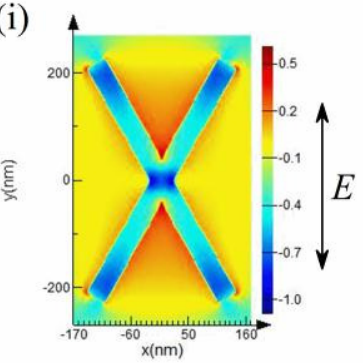

Figure S13 Electromagnetic field simulations at different angles between laser polarizations and longitudinal orientation of AgNPs. The corresponding laser polarizations are indicated by arrows. (a) and (d), (b) and (e), (c) and (f) display the enhanced electromagnetic field distributions at distances of $0 \mathrm{~nm}, 10 \mathrm{~nm}$, and $-10 \mathrm{~nm}$, respectively, away from AgNPs surface when the cross angles are fixed at $30^{\circ}$. (g)-(i) Enhanced electromagnetic field distributions at distances of $0 \mathrm{~nm}, 10 \mathrm{~nm}$, and $-10 \mathrm{~nm}$, respectively, away from AgNPs surface when the cross angles are fixed at $60^{\circ}$.

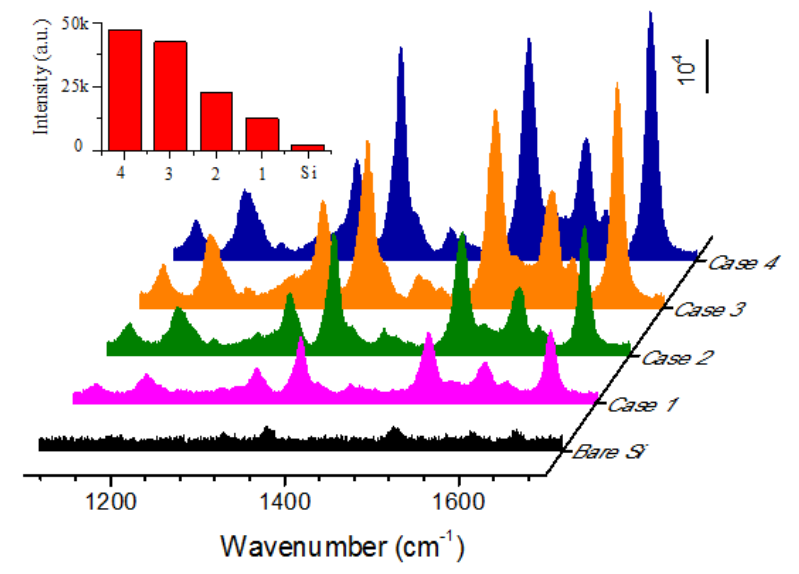

Figure S14 The comparison SRES results with different time. Case 1, Case 2, Case 3, and Case 4 represent growth time of $0 \mathrm{~h}, 5 \mathrm{~h}, 8 \mathrm{~h}$ and $10 \mathrm{~h}$, respectively. The signal on Bare Si has been magnified by 100 times. The insets are the corresponding SERS intensity of $\sim 1643 \mathrm{~cm}^{-1}$. 


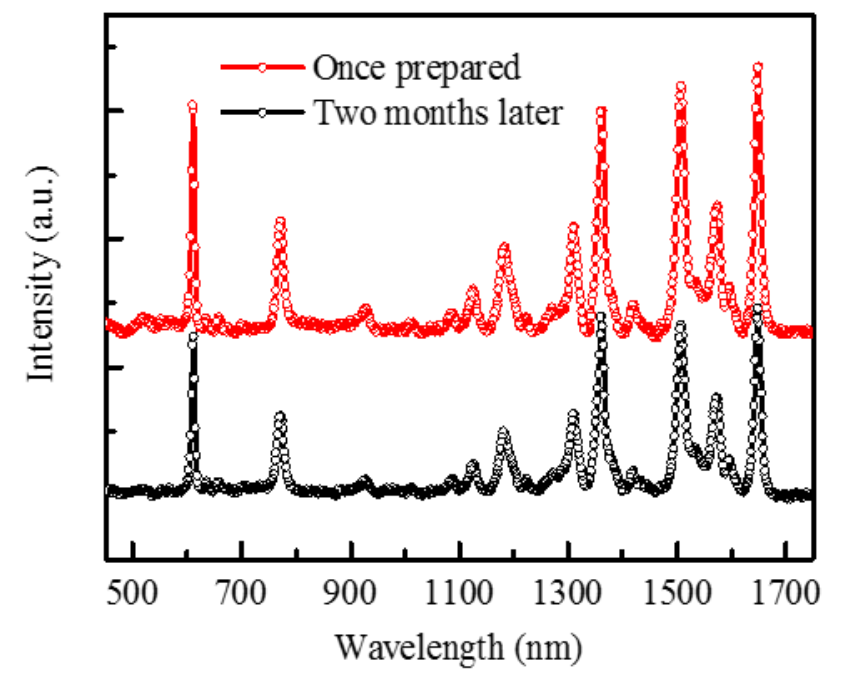

Figure S15 SERS stability experiments of AgNPs arrays. The prepared SERS substrates maintain high sensitivity in detection of R6G molecules $\left(10^{-6} \mathrm{M}\right)$ when stored in water under dark environment for two months.

(a)

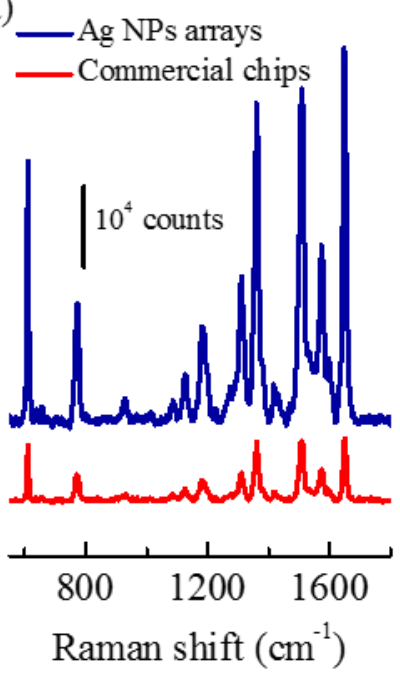

(b)

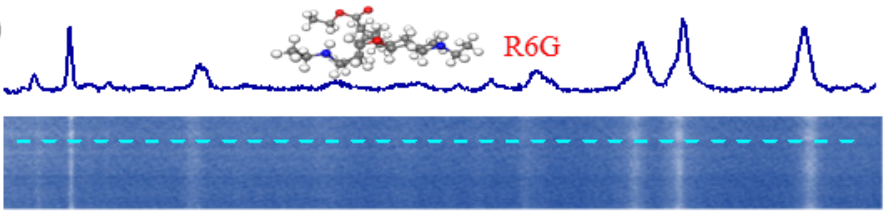

(c)

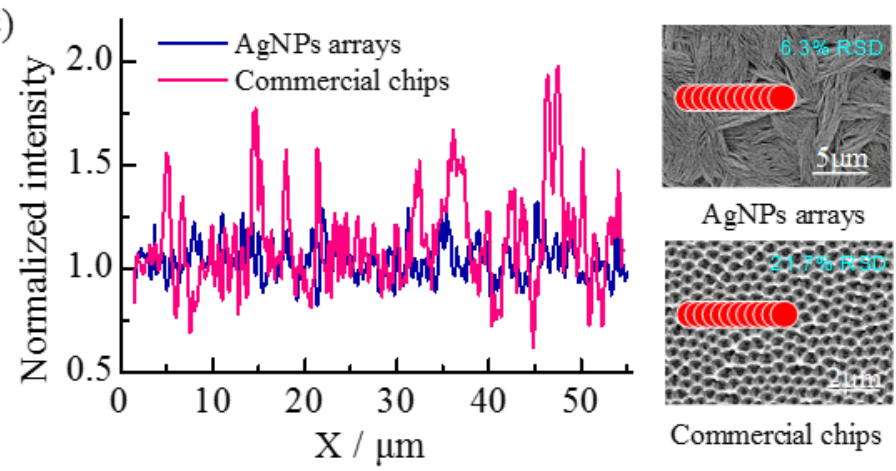

Figure S16 (a) Sensitivity of SERS performances between AgNPs arrays and commercial SERS substrate. (b) Uniformity measurements of AgNPs arrays. (c) RSD calculations of the two SERS substrates. The excitation wavelength is $633 \mathrm{~nm}$. Other form factors are the same. 


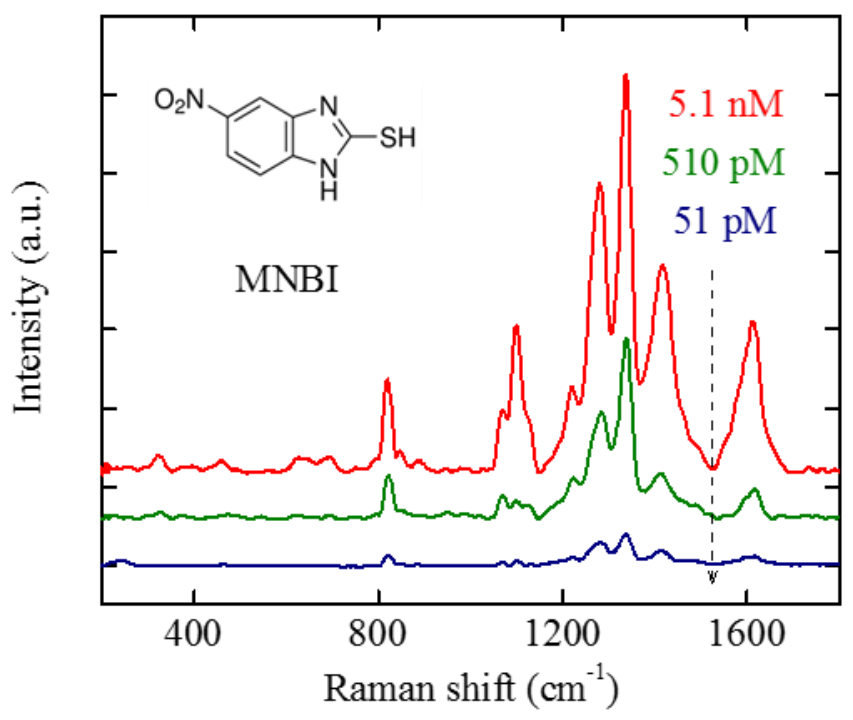

Figure S17 Trace detection of different concentration of MNBI molecules with the prepared substrate.
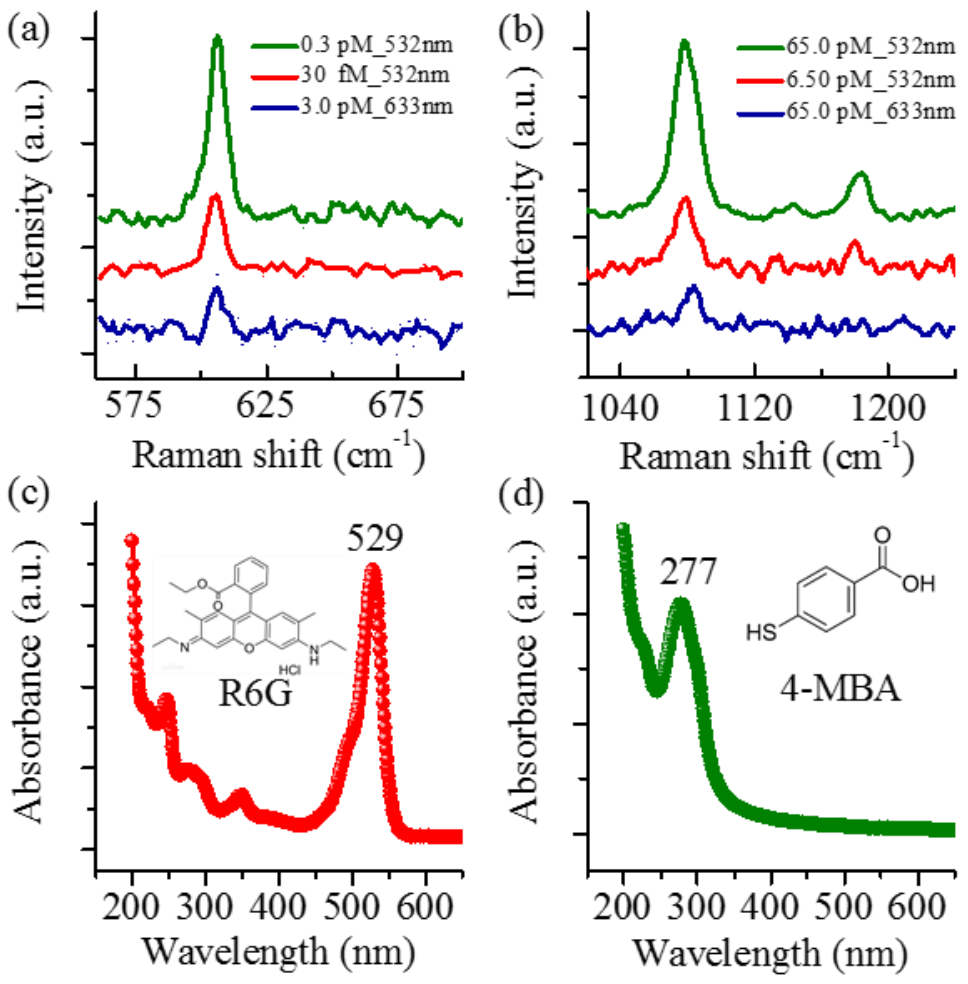

Figure S18 The resonant effects on the Raman signals of (a) R6G and (b) 4-MBA, respectively, by comparing the intensity of $532 \mathrm{~nm}$ with $633 \mathrm{~nm}$ excitation. UV-vis spectrum of (c) rhodamine 6G (R6G), and (d) 4-Mercaptobenzoic acid (4-MBA) molecules. 